Original paper

\title{
Petrology and monazite dating of the Fe-rich gneisses from Kokava (Veporic Unit, Western Carpathians, Slovakia): Devonian sediments supplied from Gondwanan sources metamorphosed in the Variscan times
}

\author{
Patrik KONEČNÝ ${ }^{*}$, Milan KOHÚT'1, Igor ROJKOVIČ², Pavol SIMAN³ \\ ${ }^{1}$ Dionýz Štúr State Institute of Geology, Mlynská dolina 1, 81704 Bratislava, Slovak Republic; patrik.konecny@geology.sk \\ ${ }^{2}$ Faculty of Natural Sciences, Comenius University, Mlynská dolina, 84215 Bratislava, Slovak Republic \\ ${ }^{3}$ Geological Institute of Slovak Academy of Sciences, Dúbravská cesta 9, 84226 Bratislava, Slovak Republic \\ * Corresponding author
}

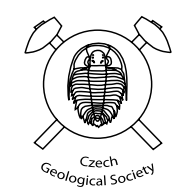

\begin{abstract}
The Fe-rich gneisses from vicinity of Kokava nad Rimavicou (Central Slovakia) were studied by electron microprobe to resolve the origin of the iron ore bodies. Whole-rock chemistry of the ironstones shows the lack of $\mathrm{CaO}, \mathrm{MgO}$ and partly elevated $\mathrm{P}_{2} \mathrm{O}_{5}$ contents. This, together with the mineral assemblage of almandine + magnetite + grunerite + ilmenite + quartz + apatite + allanite + zircon + monazite suggests an origin by metamorphism of sedimentary protolith and disproves the older idea about the formation as a skarn. The Fe-Ti oxides thermometry suggests temperatures of $360-420^{\circ} \mathrm{C}$, and the average oxygen fugacity of $\Delta \log f O_{2}$ normalized to the $\mathrm{FM} \alpha \mathrm{Q}$ of +0.13 . The reconstructed oxygen fugacity for temperatures $500-600^{\circ} \mathrm{C}$ (amphibolite facies) yields an interval of $c$. +1 to $+3 \Delta \log f O_{2}(\mathrm{FM \alpha Q})$. The presence of detrital zircons, and monazites dated in this study, reveals a participation of lithologies of the pan-African orogen. The source rocks of the Devonian ironstones sedimented probably as oolitic chamosite in lagoons.

The dated detrital monazite cores (612-400 Ma) show an affinity to source rocks formed in northern peri-Gondwana due to rifting and opening of the Rheic Ocean and separation of Avalonia and Armorica microcontinents. The majority of monazite data from the rims of detrital grains and from unzoned metamorphic grains bear an evidence of Meso-Variscan metamorphism with ages clustering between 360 and $320 \mathrm{Ma}(342 \pm 4 \mathrm{Ma}$ - weighted mean $\pm 2 \sigma)$. The younger population with elevated Th contents (18.0-20.5 wt. \%) giving the ages of $310-240 \mathrm{Ma}(279 \pm 2 \mathrm{Ma}$ - weighted mean) is connected with the collapse of Variscan orogen and/or the onset of the early Alpine continental rifting. The Alpine ages are very rare, but a few monazites and one Th- $\mathrm{U}-\mathrm{Si}$ inclusion in quartz yielded Cretaceous ages (115-85 Ma).
\end{abstract}

Keywords: Devonian metamorphosed ironstones, petrology, monazite dating, Variscan orogeny, Veporic Unit of the Western Carpathians Received: 21 January, 2011; accepted: 6 June 2011; handling editor: M. Štemprok

\section{Introduction}

Iron ores have been formed throughout the Earth's history from the Archean up to recent. Proterozoic banded iron formations (BIF) associated mostly with submarine volcanic exhalations have been reported from many localities worldwide (e.g. Floran and Papike 1978; Klein and Ladeira 2000; Mücke 2003; Sturesson 2003; Klein 2005; Mücke and Farshad 2005). From Palaeozoic times (Ordovician and Devonian) up to recent the iron ores were mostly accumulated in oolitic ferruginous sediments (Mellon 1962; Kimberley 1974; Petránek and Van Houten 1977; Sturesson et al. 1999).

The iron-rich rocks from Kokava nad Rimavicou, Central Slovakia, pertaining to the South Veporic Unit, are a part of the so-called hybrid zone (Bezák et al. 1999; Hraško et al. 2005), a region consisting of various high-grade metamorphic rocks. The genesis of the ironstones has been a matter of controversy in past decades. The first fundamental account of petrology and genesis of the Kokava ironstones was published by Šuf (1938) suggesting a skarn origin. Later, Zoubek and Nemčok (1951) pointed to a striking deficit of carbonates and rejected the skarn hypothesis. Instead, the regional metamorphism of sedimentary iron ores was thought to be the process leading to the formation of ironstones. On the contrary, Gubač (1957) agreed again with the skarn-type model. All later works interpreted this Fe-rich metamorphic formation on the basis of precise knowledge of the regional geology, mineralogy and petrology as metamorphosed oolitic ores (Zoubek and Nemčok 1951; Korikovskij et al. 1989; Bezák et al. 1999; Radvanec 2000; Kováčik 2000; Slavkay et al. 2004; Hraško et al. 2005; Siman and Janák 2005). The aim of the current paper is - based on new petrological studies and EMPA monazite dating - to further constrain the origin and evolution of the Kokava ironstones. 

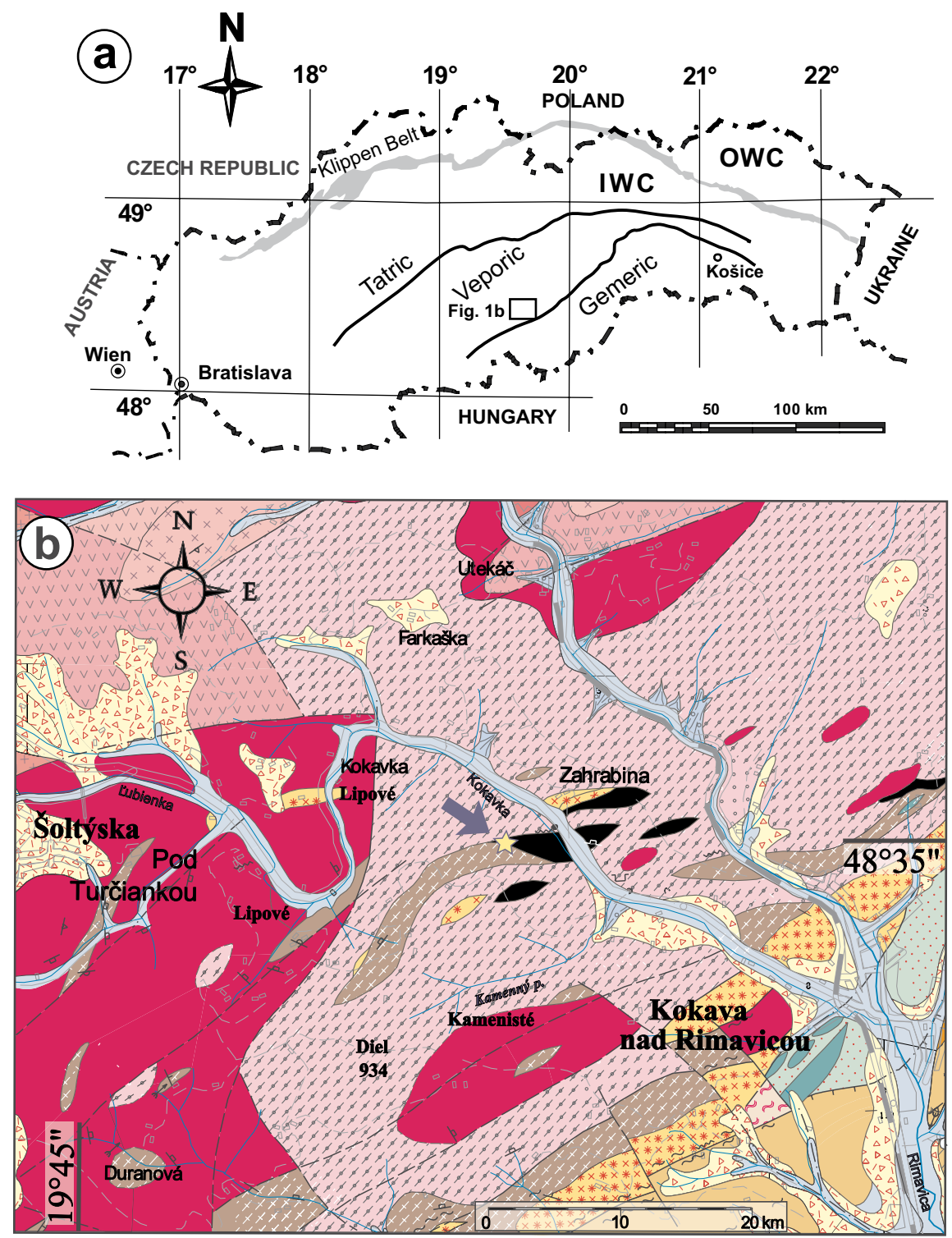

\section{Quaternary: Holocene}

fluvial \& proluvial sediments: sands, sandy soils, gravels
Pleistocene to Holocene

Fig. 1 Geological map and sample locality. a - Simplified tectonic-geological sketch of the Western Carpathians (Slovak part), displaying the principal tectonic units and position of the study area. Explanations: OWC - Outer Western Carpathians, IWC - Inner Western Carpathians; b - Geological map of the Kokava nad Rimavicou area in the southern part of the Veporic Unit (taken from the geological map 1:50 000 by Hraško et al. 2005). Arrow points to the position of the collected samples. 


\section{Geological setting and origin of Fe-rich gneisses}

The Western Carpathians are the northernmost, E-W trending branch of the Alpine orogenic belt, linked to the Eastern Alps in the west and to the Eastern Carpathians in the east. Recent structure of the Alps and the Carpathians originated from the subduction-collision (convergence) processes of the African Plate fragments (Adria-Apulia) with Eurasia after the closure of the Meliata Ocean mainly from the Cretaceous up to the present. The Western Carpathians are divided into two belts: the Outer Western Carpathians, consisting mostly of Neo-Alpine nappes, and the Inner Western Carpathians with essentially a Palaeo-Alpine structure overlain by Tertiary postnappe deposits (Fig. 1a). The Inner Western Carpathians consist of three main crustal-scale superunits which are, from north to south: the Tatric, Veporic and Gemeric and several cover-nappe systems: the Fatric, Hronic and Silica (Plašienka et al. 1997). The basement units together with their Mesozoic cover and nappe complexes were tectonically juxtaposed by a north-directed Cretaceous thrusting.

The studied rock sequence occurs in the southern part of the Veporic Unit (Fig. 1a). The Veporic Unit is the middle one of the three major south-dipping, thickskinned basement/cover imbricated units of the Western Carpathians. The Veporic basement with polyorogenic history is composed of various metamorphic and igneous rocks which reflect multistage magmatic and metamorphic episodes since the late Proterozoic to the Cretaceous (Putiš et al. 2001, 2008; Gaab et al. 2005, 2006). The Variscan HT-MP metamorphism with the concomitant widespread granitic magmatism, which heavily overprinted basement precursors and masked the polyorogenic history in the Veporic area during the Early to Late Carboniferous, is commonly accepted, whereas the Permian metamorphic event was identified only recently (Jeřábek et al. 2008). The Alpine tectono-metamorphic reworking of the Veporic Unit is a phenomenon known for more than 70 years (Zoubek 1936). Nearly all the rock complexes of the Veporic Unit were affected by an intense Alpine tectono-metamorphic reworking constrained by Ar-Ar cooling ages (Maluski et al. 1993; Dallmeyer et al. 1996; Kováćik et al. 1996; Král' et al. 1996; Janák et al. 2001). The highest $\mathrm{P}-\mathrm{T}$ conditions of Alpine metamorphism reached $600-620^{\circ} \mathrm{C}$ and $1.0-1.2 \mathrm{GPa}$ in the southeastern part of the Veporic basement (Plašienka et al. 1999; Janák et al. 2001) and greenschist-facies conditions in the metamorphosed cover sequences (Vrána 1966; Korikovsky et al. 1997; Lupták et al. 2003). However, in the north-western Veporic Unit, subduction-related eclogites recorded metamorphic conditions of c. $2.5 \mathrm{GPa}$ and $700^{\circ} \mathrm{C}$ (Janák et al. 2007), reflecting the pre-Alpine history of this unit.
The studied iron ore body is located $2.5-3 \mathrm{~km}$ WNW of Kokava nad Rimavicou on both sides of the Kokavka stream at the Hrabina locality (Fig. 1b). Five elongated, lenticular ore bodies concordant with the main structures of Variscan metamorphic rocks represent the occurrence of ironstones, known to the geological community under the name "Kokava skarn".

The main rock types were characterized as Fe-rich grunerite-almandine, or magnetite-grunerite-almandine gneisses with a low $\mathrm{Ca}$ content (Korikovskij et al. 1989). Based on mineral proportions, Kováčik (2000) described six rock types from this locality. The skarntype origin was excluded due to the lack of $\mathrm{Ca}$ and $\mathrm{Mg}$ in the studied rocks (Korikovskij et al. 1989). The wholerock geochemical composition is almost identical with chamosite oolitic ironstone formations of sedimentary Phanerozoic iron ores or to Precambrian jaspilite formations (Mücke 2003). The presence of chamosite oolites with sandstones, black quartzites and P-rich laminae suggests a shallow-sea sedimentation (Korikovskij et al. 1989; Kováčik 2000).

Fe-rich rocks formed during the multistage Variscan evolution of the Veporic Unit. The evidence for initial crustal thickening during Palaeo-Variscan event (405-400 $\mathrm{Ma}$ ) is preserved only rudimentary. After a period of stagnation, denudation and sedimentation followed the main Meso-Variscan metamorphism (370-350 Ma). It was responsible for regional metamorphic overprint and origin of granitic melts intruding metamorphic rocks. The regional cooling associated with retrograde metamorphism contributed to the infiltration of fluids during postorogenic extension and exhumation in the Late Carboniferous (Bezák et al. 1999; Hraško et al. 2005; Siman and Janák 2005).

\section{Methods}

Chemical composition of the mineral phases was studied by electron microprobe CAMECA SX 100 at the Dionýz Štúr State Institute of Geology in Bratislava. Analytical conditions were: sample current $20 \mathrm{nA}$, accelerating voltage $15 \mathrm{KV}$, beam diameter $1-10 \mu \mathrm{m}$. Due to high sensitivity to electron beam, calcite was measured with beam defocused to $15-20 \mu \mathrm{m}$. Natural and synthetic standards were used for calibration of following elements and corresponding spectral lines: albite $\left(\mathrm{Na} \mathrm{K}_{\alpha}\right)$, orthoclase $(\mathrm{K}$ $\mathrm{K}_{\alpha}$ ), wollastonite $\left(\mathrm{Si} \mathrm{K} \mathrm{K}_{\alpha}, \mathrm{Ca} \mathrm{K}_{\alpha}\right), \mathrm{Al}_{2} \mathrm{O}_{3}\left(\mathrm{Al} \mathrm{K} \mathrm{K}_{\alpha}\right), \mathrm{MgO}$ $\left(\mathrm{Mg} \mathrm{K}_{\alpha}\right), \mathrm{TiO}_{2}(\mathrm{Ti} \mathrm{K} \alpha)$, hematite $\left(\mathrm{Fe} \mathrm{K}{ }_{\alpha}\right)$, rhodonite $(\mathrm{Mn}$ $\left.\mathrm{K}_{\alpha}\right)$, chromite $\left(\mathrm{Cr} \mathrm{K}_{\alpha}\right)$, pure vanadium $\left(\mathrm{V} \mathrm{K}_{\alpha}\right), \mathrm{SrTiO}_{3}$ $\left(\mathrm{Sr} \mathrm{L}_{\alpha}\right)$, pure nickel $\left.(\mathrm{Ni} \mathrm{K})_{\alpha}\right), \mathrm{ZnS}\left(\mathrm{Zn} \mathrm{K}_{\alpha}\right), \mathrm{BaSO}_{4}(\mathrm{Ba}$ $\left.\mathrm{L}_{\alpha}\right), \mathrm{UO}_{2}\left(\mathrm{U} \mathrm{M}_{\beta}\right), \mathrm{ThO}_{2}(\mathrm{Th} \mathrm{M} \beta), \mathrm{PbS}\left(\mathrm{Pb} \mathrm{M}_{\alpha}\right), \mathrm{LaPO}_{4}$ $\left(\mathrm{La} \mathrm{L}_{\alpha}\right), \mathrm{CePO}_{4}\left(\mathrm{Ce} \mathrm{L}_{\alpha}\right), \mathrm{PrPO}_{4}\left(\mathrm{Pr} \mathrm{L}_{\beta}\right), \mathrm{NdPO}_{4}\left(\mathrm{Nd} \mathrm{L}_{\beta}\right)$, $\mathrm{SmPO}_{4}\left(\mathrm{Sm} \mathrm{L}_{\beta}\right), \mathrm{EuPO}_{4}\left(\mathrm{Eu} \mathrm{L}_{\beta}\right), \mathrm{ZrSiO}_{4}\left(\mathrm{Zr} \mathrm{L}_{\alpha}\right), \mathrm{HfO}_{2}$ 
(Hf L $\left.\mathrm{L}_{\beta}\right), \mathrm{YPO}_{4}\left(\mathrm{Y} \mathrm{L}_{\alpha}\right), \mathrm{GdPO}_{4}\left(\mathrm{Gd} \mathrm{L}_{\alpha}\right), \mathrm{TbPO}_{4}\left(\mathrm{~Tb} \mathrm{~L}_{\alpha}\right)$, $\operatorname{DyPO}_{4}\left(\mathrm{Dy} \mathrm{L}_{\beta}\right), \mathrm{HoPO}_{4}\left(\mathrm{Ho} \mathrm{L}_{\beta}\right), \mathrm{ErPO}_{4}\left(\mathrm{Er} \mathrm{L}_{\beta}\right), \mathrm{TmPO}_{4}$ (Tm L $\left.\mathrm{L}_{\alpha}\right), \mathrm{YbPO}_{4}\left(\mathrm{Yb} \mathrm{L}_{\alpha}\right), \mathrm{LuPO}_{4}\left(\mathrm{Lu} \mathrm{L}_{\beta}\right)$, GaAs $\left(\mathrm{As} \mathrm{L}_{\alpha}\right)$, apatite $\left(\mathrm{P} \mathrm{K}_{\alpha}\right), \mathrm{BaF}_{2}\left(\mathrm{~F} \mathrm{~K}_{\alpha}\right)$ and $\mathrm{NaCl}(\mathrm{Cl} \mathrm{K})_{\alpha}$. Backscattered electron (BSE) images were utilized for the study of relationships between mineral phases and for photographic documentation.

Electron probe micro-analysis (EPMA) monazite dating requires special conditions suitable for the precise measurement of trace $\mathrm{Pb}$ and $\mathrm{U}$ concentrations. Analytical conditions that compromise sufficient counting statistics with minimum deterioration of the measured spot were as follows: accelerating voltage $15 \mathrm{kV}$, sample current $85 \mathrm{nA}$, beam diameter 2-5 $\mu \mathrm{m}$. Measured elemental concentrations were corrected for the interferences, the most important being the $\mathrm{PbM}_{\alpha}-\mathrm{YL}_{\gamma}$ and $\mathrm{UM}_{\beta}-\mathrm{ThM}_{\alpha}$ overlaps. Concentrations were recalculated according to the model by Montel (1996), using own software DAMON. The calibration was checked by four monazite standards previously dated by SHRIMP with deviation about $5 \mathrm{Ma}$ from the standard ages. More details on the dating technique were published by Konečný et al. (2004) and Petrík and Konečný (2009).

The bulk-rock composition was analyzed by ICP-AES at Geoanalytical Laboratory, Dionýz Štúr State Institute of Geology, Spišská Nová Ves.

\section{Results}

\subsection{Whole-rock chemical composition of the ironstone}

Fe-rich crystalline schists show a specific chemical composition (Tab. 1). Alkalies are entirely missing and $\mathrm{CaO}$ content is extremely low. A characteristically high $\mathrm{Fe}$ content is bound to magnetite, ilmenite, almandine and grunerite. High $\mathrm{P}$ and $\mathrm{Zr}$ contents are related to high apatite, monazite and zircon contents. Increased $\mathrm{La}$ (and obviously other REE, not analyzed) and Y correspond to elevated proportions of monazite and allanite. Low $\mathrm{CO}_{2}$ suggests the rarity of carbonates, contrary to their common presence in the worldwide localities. Most $\mathrm{CaO}$ is bound in garnets.

\subsection{Mineralogical characteristics of the iron- stones}

The studied sample has a typical fine-grained equigranular texture, partly with a weak foliation that mimics the original rock bedding. The modal proportions of rockforming minerals estimated using optical microscope and BSE images are as follows: garnet (30-35 vol. \%), magnetite (20-25 vol. \%), amphibole (15-20 vol. \%), il-
Tab. 1 Whole-rock chemical composition of the studied sample Kk-3

\begin{tabular}{|c|c|c|c|}
\hline \multicolumn{4}{|c|}{ Kokava, sample Kk-3 } \\
\hline & wt. \% & & ppm \\
\hline $\mathrm{SiO}_{2}$ & 32.45 & $\mathrm{Ba}$ & $<5$ \\
\hline $\mathrm{Al}_{2} \mathrm{O}_{3}$ & 6.85 & Co & 35 \\
\hline $\mathrm{Fe}_{2} \mathrm{O}_{3}$ & 51.67 & $\mathrm{Cr}$ & 146 \\
\hline $\mathrm{TiO}_{2}$ & 2.43 & $\mathrm{Cu}$ & 15 \\
\hline $\mathrm{CaO}$ & 3.16 & $\mathrm{La}$ & 218 \\
\hline $\mathrm{MgO}$ & 0.59 & Mo & 3 \\
\hline $\mathrm{MnO}$ & 2.27 & $\mathrm{Ni}$ & 73 \\
\hline $\mathrm{P}_{2} \mathrm{O}_{5}$ & 1.05 & $\mathrm{~Pb}$ & $<4$ \\
\hline $\mathrm{Na}_{2} \mathrm{O}$ & $<0.01$ & $\mathrm{Sr}$ & 100 \\
\hline $\mathrm{K}_{2} \mathrm{O}$ & 0.01 & $\mathrm{~V}$ & 264 \\
\hline $\mathrm{H}_{2} \mathrm{O}^{-}$ & 0.19 & $\mathrm{Y}$ & 113 \\
\hline LOI & $<0.01$ & $\mathrm{Zr}$ & 4135 \\
\hline total & 100.67 & & \\
\hline TC & 0.58 & & \\
\hline TOC & 0.12 & & \\
\hline TIC & 0.46 & & \\
\hline $\mathrm{CO}_{2}$ carb. & 1.68 & & \\
\hline
\end{tabular}

Explanation: TC - total carbon, TOC - total organic carbon, TIC - total inorganic carbon, LOI - loss on ignition

menite (15-20 vol. \%) and quartz (10-15 vol. \%). Apatite and allanite reach rather high contents, up to 5 vol. \%. Biotite is subordinate $(<1$ vol. \%). Zircon and monazite were identified among the accessory minerals. Alteration products are chlorite, cerianite, barite and $\mathrm{Fe}-\mathrm{Co}-\mathrm{As}$ sulphides. Although Kováčik (2000) has subdivided the $\mathrm{Fe}$-rich rocks from the Kokava region into six mineralogically distinct groups, the studied rock sample does not fit to any of them. This documents well a wide variability of Kokava Fe-rich rocks and associated gneisses.

Ore minerals like magnetite and ilmenite are abundant. Magnetite (Fig. 2a-b) forms either isometric, mostly round grains $0.1-0.2 \mathrm{~mm}$ in size or angular to euhedral crystals. Magnetite is characterized by low $\mathrm{TiO}_{2}(0.4-0.8$ wt. \%, average 0.52 wt. $\%$ ), $\mathrm{Al}_{2} \mathrm{O}_{3}$ (average 0.25 wt. \%),

Fig. 2 Back-scattered electron (BSE) images of mineral phases. a - Rare allanite inclusion in almandine, oriented inclusions of ilmenite II in grunerite, large ilmenite I intergrown with magnetite; $\mathbf{b}$-Alteration of allanite, position of chain quartz II in grunerite, position of quartz I, magnetite with ilmenite exsolutions; c - Rounded monazite, zircon enclosed in almandine and quartz I, magnetite, ilmenite, almandine and allanite overgrowth; grunerite formed after above mentioned association in the interstitial spaces; $\mathbf{d}$ - Inclusion of monazite rimmed by apatite and then by allanite in almandine; quartz I is also included in almandine; $\mathbf{e}-$ A large monazite inclusion $(60 \mu \mathrm{m})$ enclosed in almandine, chlorite filling crack in almandine; $\mathbf{f}$ - Detrital core of Proterozoic age replaced by younger (mid-Variscan) monazite from outer boundary; g - Round monazite-apatite inclusion of a typical egg-like shape enclosed by large almandine. Note that the magnetite is younger than this inclusion; h - A Th-U-Si phase, small inclusion in quartz was dated as Cretaceous. Abbreviations used: $\mathrm{mnz}$ - monazite, alm - almandine, aln - allanite, $\mathrm{mt}$ - magnetite, ilm - ilmenite, zrn - zircon, grun - grunerite, ap - apatite and qtz - quartz. 

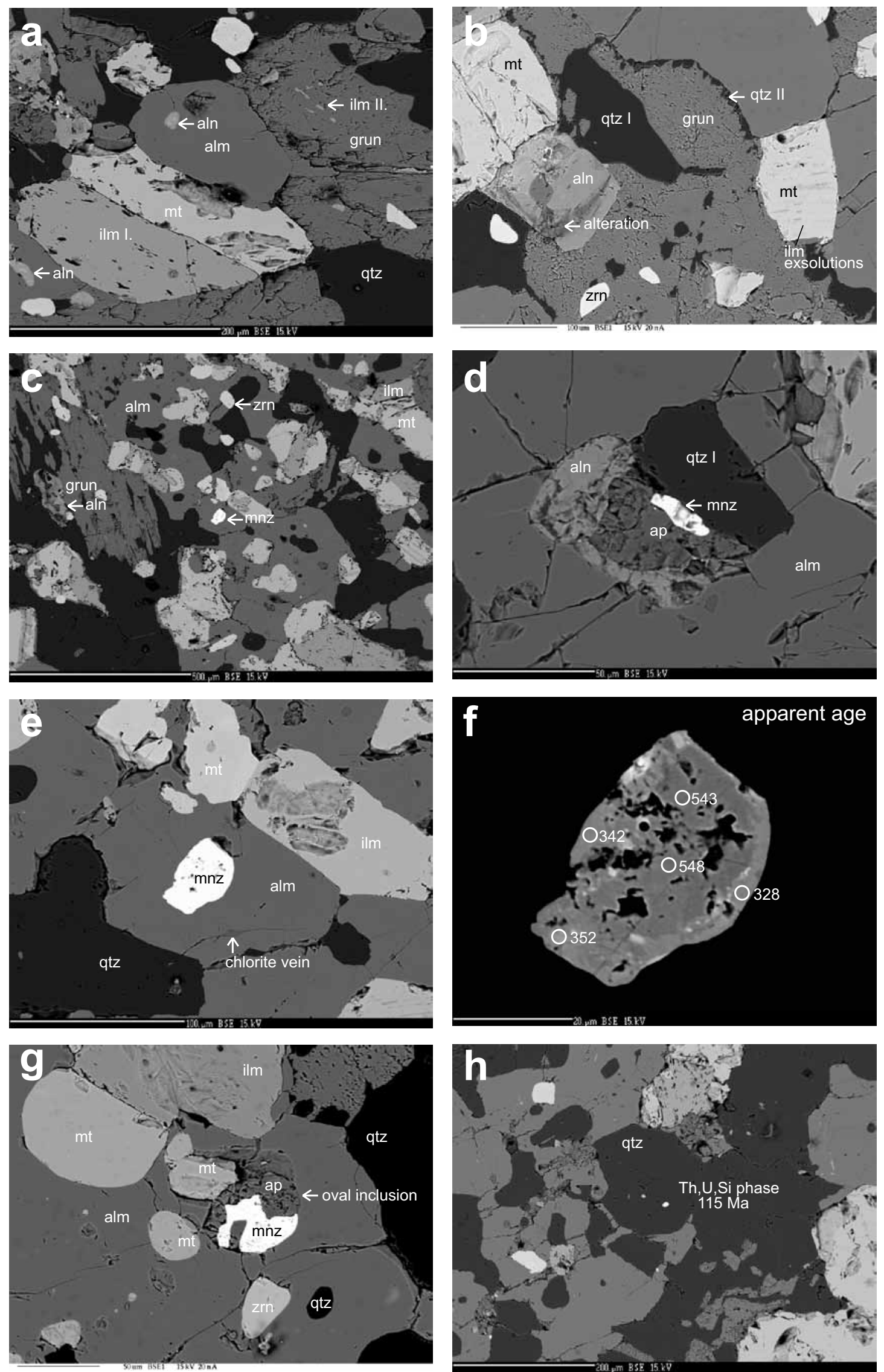
and $\mathrm{MnO}$ contents $(<0.1 \mathrm{wt} . \%)$. It is slightly enriched in $\mathrm{V}$ (average 0.22 wt. \%, seldom up to $1.1 \mathrm{wt} \%$ ). Most magnetites are free of exsolutions. Only a few contain ilmenite exsolutions in the form of tiny lamellae (Fig. 2b) or coarse isometric intergrowths. Ilmenite I (Fig. 2a-b) is fairly abundant, forming solitary crystals or intergrowths with magnetite (Fig. 2c). Increased $\mathrm{MnO}$ contents (1.82.4; average 2.23 wt. \%) correspond to slightly higher pyrophanite mole proportion $\left(\mathrm{X}_{\mathrm{pyr}} 0.042-0.053\right)$. The $\mathrm{MgO}, \mathrm{CaO}$ and $\mathrm{ZnO}$ concentrations are negligible $(<0.5$ wt. \%). Similarly to magnetite, ilmenite I is enriched in $\mathrm{V}\left(0.14\right.$ wt. $\left.\% \mathrm{~V}_{2} \mathrm{O}_{3}\right)$. Ilmenites show low ferric iron concentrations corresponding to a low hematite mole fraction $\left(X_{\text {hem }}\right.$ 0.002-0.015). The highest concentrations were observed in the exsolution lamellae $\left(\mathrm{X}_{\text {hem }} 0.045-0.067\right)$ in magnetite. Almandines (Tab. 2) form irregular or equant grains homogeneously distributed in the rock. Grunerite is mostly prismatic with spindle-like crystals, often forming local fans or sheaves. Allanite is ochre brown to orange-brown in transmission light, indicating a decomposition or metamictization. It forms columnar crystals mostly localized in the interstitial spaces among almandine, magnetite and ilmenite crystals. Allanite inclusions in almandine are very rare (Fig. 2a). Some allanites contain monazite or monazite-apatite intergrowths in the core (Fig. 2d). Patchy zoning is visible in BSE images, mostly as alternating pale and darker irregular zones. Monazite is present in accessory amount (Fig. 2c). mostly forming small rounded isometric crystals up to $25-30 \mu \mathrm{m}$ in size (rarely up to $50 \mu \mathrm{m}$ ), often included in almandine. Less frequently it is also surrounded by allanite and both included in almandine. Monazite is never enclosed in quartz or grunerite. On the contrary, allanite inclusions in quartz are very common. Some allanites contain monazite \pm apatite in their cores.

According to mutual mineral relationships in BSE images and mineral inclusions, we determined the crystallization sequence of the Fe-rich rocks. Minerals with oval shape like zircon, monazite, monazite intergrowths with apatite, often found as inclusions in younger minerals (magnetite, ilmenite and almandine), are regarded as the earlier mineral phases. The oval shape of inclusions indicates their likely sedimentary origin. At a later stage took place a joint crystallization of garnet, ilmenite I, magnetite, and quartz I, which is supported by observations of inclusion relationships or intergrowths. The role of allanite is ambiguous. Allanite associated with monazite and apatite, rounded together with these minerals and included in almandine, formed most likely during sedimentation. On the other hand, euhedral allanites hosted in quartz or grunerite are of a metamorphic origin. Quartz I (Fig. 2d) can easily be recognized by the presence of numerous tiny oval crystals included in garnet, thus resembling a sieve-like texture. The final stage corresponded to crystallization of grunerite and quartz II. Evidence for the latest formation is the absence of grunerite inclusions in ilmenite, magnetite or almandine. Some larger euhedral allanite crystals partially enclosed in the swarms of needle-like grunerites indicate younger growth of grunerite. Grunerite fills intergranular spaces among older minerals (Fig. 2c). It is often intergrown with later quartz II. In some places, polycrystalline aggregates of grunerite are rimmed with chains of triangular quartz crystals probably indicating a concomitant or later growth of quartz II after grunerite (Fig. 2b). In other places grunerite forms euhedral crystals surrounded by quartz II. Tiny ilmenite grains accompanied crystallization of grunerite and quartz II (Fig. 2a). Ilmenite II is very rare and is found as tiny ilmenite needles in grunerite with parallel orientation to c-axis of the host grunerite (Fig. 2a). Similar tiny needles, but without preferred orientation, are seldom scattered in quartz II. Biotite also belongs to the late-stage mineral assemblage. It is unusually rare and was identified only as small inclusions in grunerite and quartz II, indicating a common origin.

Large euhedral grunerite surrounded by biotite matrix and younger fibrous anhedral grunerite replacing biotite was found by Kováčik (2000) in neighbouring gneisses. Such mineral relations suggest that large euhedral grunerite crystallized before biotite. Our observations from the Kokava Fe-rich rock (presence of tiny biotites included in grunerite) indicate just an opposite relationship.

Chlorite represents an alteration product, probably of grunerite. Chloritization is only locally developed, and chlorite forms fillings of small veins (Fig. 2e). Cerianite (Tab. 3) is also considered to be an alteration product; namely a result of allanite decomposition. It fills cracks in allanite or penetrates along cracks into neighbouring minerals. More advanced decomposition leads to the formation of thick cerianite rims around allanite. Rarely is allanite replaced by barite and tiny sulphides.

\subsection{Fe-Ti thermometry and oxygen fugacity}

The Fe-Ti oxides form complicated solid solutions with different types of ordering (long-range convergent and nonconvergent order or short-range order), which can be used in geothermobarometry. The geothermometer/ oxygen barometer QUILF by Frost et al. (1988), Frost and Lindsley (1992) and Lindsley and Frost (1992) was used for $T-\mathrm{fO}_{2}$ estimates. QUILF guarantees internally consistent results for oxygen geobarometry between $\mathrm{Fe}-$ Ti oxides and coexisting silicate phases.

For $T-f \mathrm{O}_{2}$ calculations were selected only analyses from coexisting magnetite-ilmenite I pairs (ilmenite exsolution lamellae in magnetite or mutual intergrowths). Ilmenite II could not be used because of the missing magnetite counterpart. The results show that Kokava Fe-rich 
Tab. 2 Electron microprobe analyses of grunerite, almandine and biotite

\begin{tabular}{|c|c|c|c|c|c|c|c|c|c|c|c|c|}
\hline & \multicolumn{3}{|c|}{ amphibole - grunerite } & \multicolumn{8}{|c|}{ garnet - almandine } & \multirow{3}{*}{$\begin{array}{c}\text { biotite } \\
7 \\
\text { incl. in } \mathrm{Mt}\end{array}$} \\
\hline & 1a & $1 \mathrm{~b}$ & 2 & & $3 a$ & $3 b$ & $4 a$ & $4 b$ & 5 & 6 & & \\
\hline & core & rim & lamellar & & core & & core & & core & incl. in $\mathrm{Mt}$ & & \\
\hline $\mathrm{SiO}_{2}$ & 49.99 & 50.31 & 50.15 & $\mathrm{SiO}_{2}$ & 36.970 & 37.028 & 36.556 & 36.977 & 36.624 & 36.189 & $\mathrm{SiO}_{2}$ & 33.93 \\
\hline $\mathrm{TiO}_{2}$ & 0.02 & 0.02 & 0.01 & $\mathrm{TiO}_{2}$ & 0.114 & 0.021 & 0.008 & 0.032 & 0.022 & 0.071 & $\mathrm{TiO}_{2}$ & 2.80 \\
\hline $\mathrm{Al}_{2} \mathrm{O}_{3}$ & 0.02 & 0.22 & 0.18 & $\mathrm{Al}_{2} \mathrm{O}_{3}$ & 19.926 & 20.424 & 20.354 & 20.415 & 20.575 & 20.090 & $\mathrm{Al}_{2} \mathrm{O}_{3}$ & 13.96 \\
\hline $\mathrm{Cr}_{2} \mathrm{O}_{3}$ & 0.04 & 0.01 & 0 & $\mathrm{Cr}_{2} \mathrm{O}_{3}$ & 0 & 0 & 0 & 0 & 0.013 & 0.019 & $\mathrm{Cr}_{2} \mathrm{O}_{3}$ & 0.01 \\
\hline $\mathrm{FeO}$ & 40.2 & 39.94 & 40.19 & $\mathrm{FeO}$ & 30.930 & 33.472 & 30.210 & 31.170 & 33.958 & 33.078 & $\mathrm{FeO}$ & 32.26 \\
\hline $\mathrm{MnO}$ & 1.11 & 1.22 & 1.19 & $\mathrm{MnO}$ & 8.181 & 7.201 & 8.151 & 7.305 & 7.283 & 7.529 & $\mathrm{MnO}$ & 0.13 \\
\hline $\mathrm{NiO}$ & & & 0.03 & $\mathrm{NiO}$ & 0.010 & 0.000 & 0.000 & 0.012 & 0 & 0 & $\mathrm{NiO}$ & 0 \\
\hline $\mathrm{MgO}$ & 5.31 & 5.27 & 5.34 & $\mathrm{MgO}$ & 0.893 & 0.387 & 0.928 & 0.536 & 0.446 & 0.382 & $\mathrm{MgO}$ & 3.31 \\
\hline $\mathrm{CaO}$ & 0.23 & 0.16 & 0.17 & $\mathrm{CaO}$ & 3.611 & 1.902 & 3.430 & 3.486 & 1.785 & 3.249 & $\mathrm{CaO}$ & 0.04 \\
\hline $\mathrm{Na}_{2} \mathrm{O}$ & 0.08 & 0.06 & 0.05 & $\mathrm{Na}_{2} \mathrm{O}$ & 0.069 & 0.030 & 0.082 & 0.055 & 0.063 & 0.082 & $\mathrm{Na}_{2} \mathrm{O}$ & 0.07 \\
\hline $\mathrm{K}_{2} \mathrm{O}$ & 0 & 0 & & $\mathrm{~K}_{2} \mathrm{O}$ & 0 & 0 & 0 & 0 & 0.002 & 0 & $\mathrm{~K}_{2} \mathrm{O}$ & 8.39 \\
\hline $\mathrm{F}$ & 0.08 & 0.05 & 0 & $\mathrm{~F}$ & & & & & 0.000 & 0.082 & $\mathrm{~F}$ & 0.11 \\
\hline $\mathrm{Cl}$ & 0.01 & 0.02 & 0.02 & $\mathrm{Cl}$ & & & & & 0.023 & 0.013 & $\mathrm{Cl}$ & 0.17 \\
\hline Total & 97.05 & 97.27 & 97.33 & Total & 100.703 & 100.463 & 99.718 & 99.988 & 100.793 & 100.784 & Total & 95.18 \\
\hline$\overline{\mathrm{TSi}}$ & 8.082 & 8.11 & 8.077 & $\overline{\mathrm{TSi}}$ & 2.998 & 3.027 & 2.987 & 3.020 & 2.986 & 4.039 & $\mathrm{Si}$ & 5.813 \\
\hline TAl & 0 & 0 & 0 & TAl & 0.002 & 0 & 0.013 & 0.000 & 0.014 & 0.000 & $\mathrm{Al}^{\mathrm{IV}}$ & 2.187 \\
\hline $\mathrm{TFe}^{3+}$ & 0 & 0 & 0 & Sum_T & 3.000 & 3.027 & 3.000 & 3.020 & 3.000 & 4.039 & $\mathrm{Al}^{\mathrm{VI}}$ & 0.629 \\
\hline TTi & 0 & 0 & 0 & $\mathrm{Al}^{\mathrm{VI}}$ & 1.901 & 1.966 & 1.945 & 1.964 & 1.961 & 1.512 & $\mathrm{Ti}$ & 0.361 \\
\hline Sum_T & 8.082 & 8.11 & 8.077 & $\mathrm{Fe}^{3+}$ & 0.105 & 0.114 & 0.103 & 0.106 & 0.116 & 0.088 & $\mathrm{Fe}^{3+}$ & 0.000 \\
\hline CAl & 0.004 & 0.042 & 0.034 & $\mathrm{Ti}$ & 0.007 & 0.001 & 0.000 & 0.002 & 0.001 & 0.003 & $\mathrm{Fe}^{2+}$ & 4.622 \\
\hline $\mathrm{CCr}$ & 0.005 & 0.001 & 0 & $\mathrm{Cr}$ & 0 & 0 & 0 & 0 & 0 & 0.001 & $\mathrm{Cr}$ & 0.001 \\
\hline $\mathrm{CFe}^{3+}$ & 0 & 0 & 0 & Sum_A & 2.013 & 2.082 & 2.049 & 2.072 & 2.078 & 1.605 & $\mathrm{Mn}$ & 0.019 \\
\hline $\mathrm{CTi}$ & 0.002 & 0.002 & 0.001 & $\mathrm{Fe}^{2+}$ & 1.993 & 2.174 & 1.961 & 2.023 & 2.199 & 1.680 & $\mathrm{Mg}$ & 0.845 \\
\hline $\mathrm{CMg}$ & 1.280 & 1.266 & 1.282 & $\mathrm{Mg}$ & 0.108 & 0.047 & 0.113 & 0.065 & 0.054 & 0.036 & $\mathrm{Ba}$ & 0.000 \\
\hline $\mathrm{CFe}^{2+}$ & 3.709 & 3.688 & 3.682 & $\mathrm{Mn}$ & 0.562 & 0.499 & 0.564 & 0.505 & 0.503 & 0.408 & $\mathrm{Ca}$ & 0.007 \\
\hline $\mathrm{CMn}$ & 0 & 0 & 0 & $\mathrm{Ca}$ & 0.314 & 0.167 & 0.300 & 0.305 & 0.156 & 0.222 & $\mathrm{Na}$ & 0.023 \\
\hline $\mathrm{CCa}$ & 0 & 0 & 0 & $\mathrm{Na}$ & 0.011 & 0.005 & 0.013 & 0.009 & 0.010 & 0.010 & $\mathrm{~K}$ & 1.834 \\
\hline Sum_C & 5.000 & 5.000 & 5.000 & Sum_B & 2.987 & 2.891 & 2.951 & 2.907 & 2.922 & 2.356 & Cations & 16.341 \\
\hline $\mathrm{BMg}$ & 0 & 0 & 0 & Sum_cat & 8.000 & 8.000 & 8.000 & 8.000 & 8.000 & 8.000 & $\mathrm{CF}$ & 0.119 \\
\hline $\mathrm{BFe}^{2+}$ & 1.726 & 1.696 & 1.731 & Alm & 67.1 & 75.9 & 67.1 & 69.8 & 75.3 & 71.6 & $\mathrm{CCl}$ & 0.098 \\
\hline BMn & 0.152 & 0.167 & 0.162 & And & 4.6 & 5.5 & 3.8 & 5.5 & 5.6 & 5.7 & & \\
\hline $\mathrm{BCa}$ & 0.040 & 0.028 & 0.029 & Grs & 5.9 & 0.3 & 6.3 & 5.0 & 0.0 & 3.8 & & \\
\hline $\mathrm{BNa}$ & 0.025 & 0.019 & 0.016 & Prp & 3.6 & 1.6 & 3.8 & 2.3 & 1.9 & 1.6 & & \\
\hline Sum_B & 1.943 & 1.909 & 1.938 & Sps & 18.8 & 17.2 & 19.0 & 17.4 & 17.2 & 17.4 & & \\
\hline $\mathrm{ACa}$ & 0 & 0 & 0 & Uv & 0 & 0 & 0 & 0 & 0 & 0.1 & & \\
\hline $\mathrm{ANa}$ & 0 & 0 & 0 & & & & & & & & & \\
\hline $\mathrm{AK}$ & 0 & 0 & 0 & & & & & & & & & \\
\hline Sum_A & 0 & 0 & 0 & & & & & & & & & \\
\hline Sum_cat & 15.025 & 15.019 & 15.016 & & & & & & & & & \\
\hline $\mathrm{CCl}$ & 0.003 & 0.005 & 0.005 & & & & & & & & & \\
\hline $\mathrm{CF}$ & 0.041 & 0.025 & 0 & & & & & & & & & \\
\hline Sum_oxy & 23.101 & 23.143 & 23.103 & & & & & & & & & \\
\hline
\end{tabular}

Grunerites are fairly homogeneous in compopsition across the grains. Cations calculated using 15NK model with negligible ferric iron.

Almandines are weakly zoned with cores enriched in Fe. Recalculation based on 12 oxygens and with $\mathrm{Fe}^{2+} / \mathrm{Fe}^{3+}$ estimated assuming full site occupancy.

Biotite is iron rich. Mineral formula was obtained by normalization on the basis of $24(\mathrm{O}, \mathrm{OH}, \mathrm{F})$.

gneisses equilibrated under temperatures of $300-400^{\circ} \mathrm{C}$ and a fugacity regime delimited by $\mathrm{FH} \alpha \mathrm{Q}$ and IM oxygen buffers (Tabs 4-5, Fig. 3). At higher temperatures of $c$. $400^{\circ} \mathrm{C}$, the oxygen fugacity values cluster around $\mathrm{FH} \alpha \mathrm{Q}$ buffer and with the dropping temperature they shift to relatively reduced conditions. Within the temperature interval of $360-420^{\circ} \mathrm{C}$, the average oxygen fugacity $\Delta \log$ $\mathrm{fO}_{2}$ normalized to the FM $\alpha \mathrm{Q}$ buffer is +0.13 . 
Tab. 3 Electron microprobe analyses of alteration pseudomorphs after allanite

\begin{tabular}{|c|c|c|c|c|c|c|c|c|c|c|c|c|}
\hline & & & lteratiol & oducts & allanit & iixture & orencit & oethite & halloys & & & cerianite \\
\hline & $1 \mathrm{a}$ & $1 b$ & $1 \mathrm{c}$ & $2 a$ & $2 b$ & $2 c$ & $3 a$ & $3 b$ & $3 c$ & 4 & 5 & in vein \\
\hline $\mathrm{SiO}_{2}$ & 4.25 & 4.36 & 10.28 & 8.15 & 7.85 & 12.18 & 14.27 & 18.57 & 32.28 & 35.92 & 6.46 & 0.50 \\
\hline $\mathrm{TiO}_{2}$ & & & & 1.26 & 1.20 & 1.11 & 0.92 & 0.79 & 0.59 & 0.28 & 0.46 & \\
\hline $\mathrm{Al}_{2} \mathrm{O}_{3}$ & 1.64 & 2.05 & 6.50 & 8.99 & 8.77 & 10.21 & 11.54 & 17.19 & 26.58 & 30.87 & 3.85 & 0.22 \\
\hline $\mathrm{Cr}_{2} \mathrm{O}_{3}$ & & & & & & & & & & & 0.00 & \\
\hline $\mathrm{FeO}$ & 50.16 & 62.75 & 59.27 & 59.95 & 60.13 & 51.21 & 47.62 & 47.62 & 30.83 & 22.99 & 64.63 & 3.77 \\
\hline $\mathrm{MnO}$ & & & & 0.17 & 0.15 & 0.28 & 0.36 & 0.08 & 0.14 & 0.25 & 0.05 & \\
\hline $\mathrm{NiO}$ & & & & & & & & & & & 0.03 & \\
\hline $\mathrm{MgO}$ & & & & & & & & & & & 0.00 & \\
\hline $\mathrm{CaO}$ & 1.06 & 2.15 & 0.27 & 0.41 & 0.26 & 1.60 & 1.90 & 0.09 & 0.30 & 0.18 & 0.06 & 1.72 \\
\hline $\mathrm{Na}_{2} \mathrm{O}$ & & & & & & & & & & & 0.10 & \\
\hline $\mathrm{K}_{2} \mathrm{O}$ & & & & & & & & & & & 0.00 & \\
\hline $\mathrm{F}$ & & & & 0.07 & 0.03 & 0.02 & 0.12 & 0.08 & 0.17 & 0.12 & 0.08 & \\
\hline $\mathrm{Cl}$ & & & & 0.07 & 0.08 & 0.08 & 0.09 & 0.17 & 0.09 & 0.06 & 0.03 & \\
\hline $\mathrm{P}_{2} \mathrm{O}_{5}$ & 10.32 & 7.72 & 1.74 & 0.33 & 0.36 & 0.25 & 0.21 & 0.23 & 0.10 & 0.08 & & 0.58 \\
\hline $\mathrm{As}_{2} \mathrm{O}_{5}$ & 0.19 & 0.08 & 0.23 & & & & & & & & & 0.19 \\
\hline $\mathrm{ThO}_{2}$ & 9.90 & 5.68 & 3.85 & 5.29 & 5.36 & 5.18 & 4.51 & 3.29 & 1.83 & 0.92 & & 9.51 \\
\hline $\mathrm{UO}_{2}$ & 0.12 & 0.06 & 0.01 & & & & & & & & & 0.17 \\
\hline $\mathrm{PbO}$ & 0.11 & 0.05 & 0.05 & & & & & & & & & 0 \\
\hline $\mathrm{Y}_{2} \mathrm{O}_{3}$ & 0.05 & 0 & 0 & 0 & 0.04 & 0 & 0.05 & 0.03 & 0 & 0 & & 0.11 \\
\hline $\mathrm{La}_{2} \mathrm{O}_{3}$ & 1.90 & 0.77 & 0.01 & 0.22 & 0.19 & 0.97 & 0.99 & 0.02 & 0.02 & 0 & & 13.56 \\
\hline $\mathrm{Ce}_{2} \mathrm{O}_{3}$ & 5.16 & 2.22 & 0.78 & 0.48 & 0.30 & 2.12 & 2.57 & 0.08 & 0.12 & 0.06 & & 35.17 \\
\hline $\mathrm{Pr}_{2} \mathrm{O}_{3}$ & 0.48 & 0.26 & 0 & 0 & 0 & 0.29 & 0.18 & 0.01 & 0 & 0 & & 3.79 \\
\hline $\mathrm{Nd}_{2} \mathrm{O}_{3}$ & 2.17 & 0.67 & 0 & 0.30 & 0.18 & 0.77 & 1.20 & 0.09 & 0.08 & 0 & & 12.34 \\
\hline $\mathrm{Sm}_{2} \mathrm{O}_{3}$ & 0.34 & 0.34 & 0.01 & 0.22 & 0.12 & 0.10 & 0.20 & 0.07 & 0.03 & 0 & & 1.89 \\
\hline $\mathrm{Eu}_{2} \mathrm{O}_{3}$ & 1.19 & 1.62 & 1.70 & & & & & & & & & 0.03 \\
\hline $\mathrm{Gd}_{2} \mathrm{O}_{3}$ & 0.35 & 0.17 & 0.04 & 0.11 & 0 & 0.11 & 0.10 & 0 & 0.04 & 0.11 & & 1.52 \\
\hline $\mathrm{Tb}_{2} \mathrm{O}_{3}$ & 0.21 & 0.24 & 0.18 & & & & & & & & & 0 \\
\hline $\mathrm{Dy}_{2} \mathrm{O}_{3}$ & 0 & 0 & 0 & & & & & & & & & 0.06 \\
\hline $\mathrm{Ho}_{2} \mathrm{O}_{3}$ & 0.03 & 0.15 & 0.08 & & & & & & & & & 0.16 \\
\hline $\mathrm{Er}_{2} \mathrm{O}_{3}$ & 0 & 0 & 0.01 & & & & & & & & & 0 \\
\hline $\mathrm{Tm}_{2} \mathrm{O}_{3}$ & 0.02 & 0 & 0 & & & & & & & & & 0 \\
\hline $\mathrm{Yb}_{2} \mathrm{O}_{3}$ & 0 & 0.10 & 0.03 & 0 & 0.08 & 0.13 & 0 & 0.15 & 0.12 & 0 & & 0.04 \\
\hline $\mathrm{Lu}_{2} \mathrm{O}_{3}$ & 0 & 0 & 0.20 & 0 & 0 & 0.17 & 0 & 0 & 0 & 0.21 & & 0.03 \\
\hline $\mathrm{SO}_{2}$ & & & & 0.47 & 0.47 & 0.19 & 0.32 & 0.50 & 0.27 & 0.31 & & \\
\hline $\mathrm{SrO}$ & & & & 0.03 & 0.02 & 0.03 & 0 & 0.04 & 0 & 0 & & \\
\hline Total & 89.55 & 91.35 & 85.17 & 86.52 & 85.58 & 86.98 & 87.15 & 89.09 & 93.57 & 92.35 & 75.74 & 85.25 \\
\hline
\end{tabular}

The same letters denote a single allanite grain. Allanites are altered to a mixture of florencite, goethite and halloysite.

Florencite and goethite prevail over the halloysite in allanite 1a-c. Note phosphorus excess.

Variable proportions of goethite and halloysite show allanites 2-4.

Allanite 5 is a pseudomorph almost completely formed by Fe oxide, goethite with subsidiary halloysite content.

The REE realeased from structure are deposided as cerianite in veins within allanite or, more often, in external cracks in surrounding minerals.

\subsection{Electron-microprobe monazite dating}

We dated various types of monazites of different shapes and dimensions. Monazites included in almandine, in oval inclusions with apatite, monazites from allanite cores. Most monazites are either zoned or unzoned. Composition of monazites varies widely, e.g. Th ranges from 2.1 to $31 \mathrm{wt}$ \%. Apparent ages plotted on histograms revealed four main populations: Late Proterozoic to Ordovician (further referred to as pre-Variscan for simplicity), Carboniferous, Permian and Alpine (Fig. 4a, Tabs 6-7).
Pre-Variscan ages range from 473 to $675 \mathrm{Ma}$, forming a broad inhomogeneous group, which is typical of detrital origin (Fig. 4b). Another evidence for detrital origin is the fact that each of the individual monazites yields similar apparent ages. The pre-Variscan grains are mostly low in Th.

Another group of apparent ages forms a single peak with nearly Gaussian distribution (Fig. 4c). The resulting age for the monazites falling into the time interval $320-400 \mathrm{Ma}$ is Middle Carboniferous, $342 \pm 4 \mathrm{Ma}(2 \sigma)$. The presence of the youngest detrital monazite dated at 


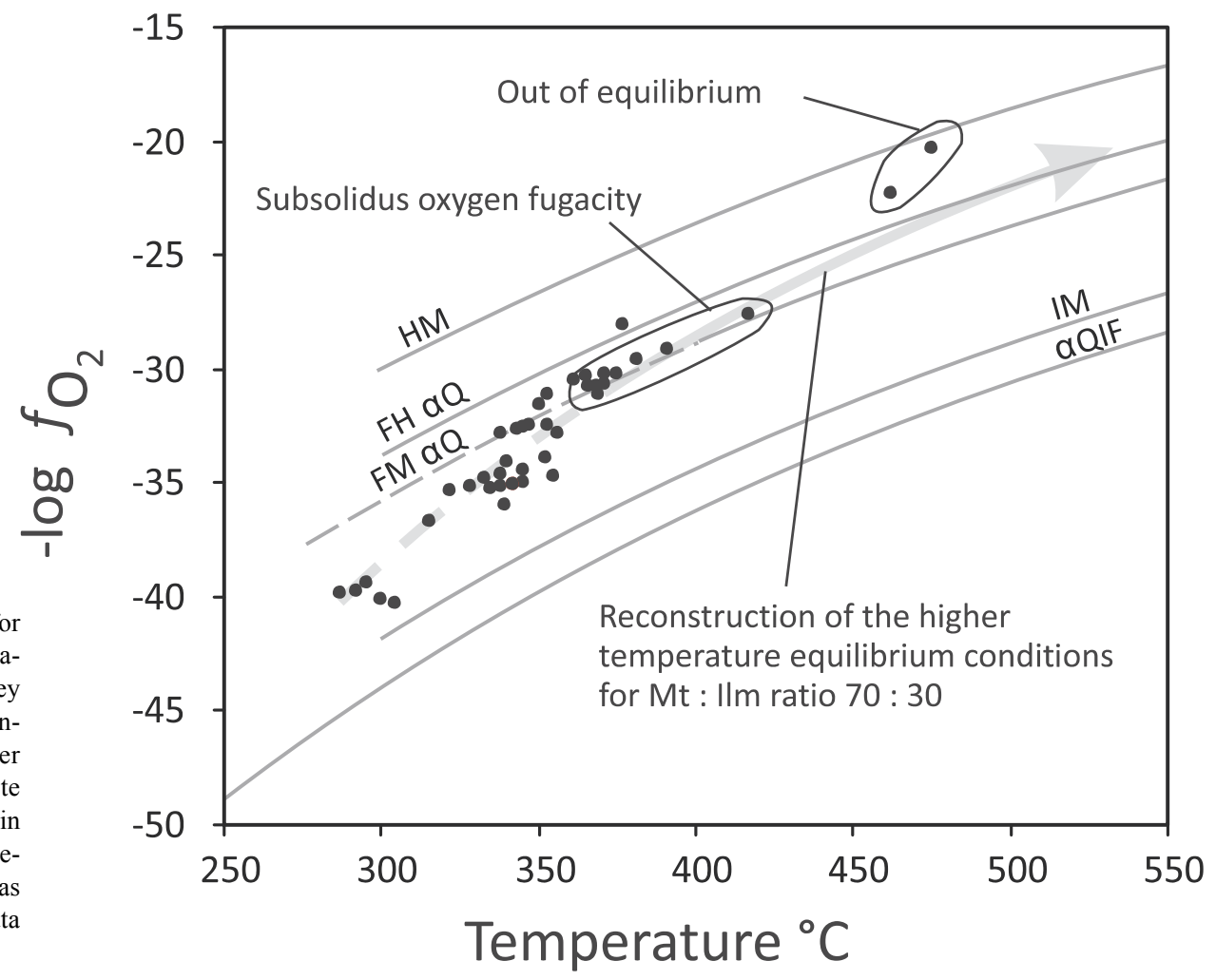

Fig. 3 Oxygen fugacity estimates for the Fe-Ti oxides. Solid lines are various types of oxygen buffers. Grey arrow denotes the direction of reconstructed oxygen fugacity at higher temperatures for magnetite-ilmenite ratio $70: 30$. At c. $500-600^{\circ} \mathrm{C}$, i.e. in course of the amphibolite-facies metamorphism, the oxygen fugacity was likely above the FM $\alpha \mathrm{Q}$ buffer. Data are given in Tabs $4-5$.

$473 \pm 14 \mathrm{Ma}$ (two analyses in the same monazite core) suggests that sedimentation of the Kokava Formation took place after the Early Ordovician but before the midCarboniferous.

Age populations and type of monazite do not correlate. Some large monazites are zoned with a dark core (BSE) and light irregular rim. Monazite dating brought evidence that the cores share Late Proterozoic-Ordovician ages whereas the rims were formed during Carboniferous (Fig. 2e-f). These zoned monazites are often included in almandine as rounded grains. It appears that the replacement of older, detrital monazites occurred only after the formation of garnet. Some small monazite inclusions in almandine are either of pre-Variscan or Carboniferous age, perhaps reflecting the variable ability of fluids to react with the old monazites. Small former detrital grains were completely replaced by Carboniferous metamorphic monazites. Monazites were enriched in Th during Variscan metamorphism.

Younger monazite population (320-240 Ma) was recorded in a few grains only (Fig. 4d). The mean age is Permian (279 \pm 2 Ma). Permian monazites (rich in Th) also occur as inclusions in garnet and show effects of interaction with the post-Variscan fluids.

The youngest, Alpine ages are found only rarely (Fig. 4a). Two ages, $84 \pm 9 \mathrm{Ma}$ and $107 \pm 5 \mathrm{Ma}$, were recorded in light rims of two monazite grains. An age $115 \pm 1 \mathrm{Ma}$ was measured in one grain of a Th-U-Si phase included in quartz (Fig. 2h). It is not possible to demonstrate con- fidently the young age population but these Cretaceous ages found in compositionally different monazites and one Th-Si phase can hint to an Alpine metamorphic overprint.

\section{Discussion}

\subsection{Temperature conditions and oxygen fugacity during re-equilibration of $\mathrm{Fe}-\mathrm{Ti}$ oxides}

The temperatures below $420^{\circ} \mathrm{C}$ calculated from the exchange reactions between cubic and rhombohedral Fe-Ti phases continue into the field of subsolidus re-equilibration. Buddington and Lindsley (1964) reported a more effective re-equilibration in $\mathrm{Fe}-\mathrm{Ti}$ oxides than between silicate phases. Combination of three processes results in re-equilibration: exchange between oxides and silicates, intraoxide and interoxide re-equilibration. Magnetites and ilmenites may react by exchange reactions with numerous silicates, e.g. olivine, orthopyroxene, augite, pigeonite and quartz. Lindsley and Frost (1992) studied 16 such reaction types. Due to the intraoxide re-equilibration, the solid solution breaks down to two phases. It is shown by exsolution of ilmenite lamellae into magnetite. The interoxide re-equilibration cannot be petrographically proved since the composition of both coexisting phases changed gradually with the decreasing temperature. Effects of 


\begin{tabular}{|c|c|c|c|c|c|c|c|c|c|c|c|c|c|c|c|c|c|c|c|c|c|c|c|c|c|c|}
\hline$\tilde{N}$ & & $=$ & $\bar{n}$ & $\tilde{y}$ & $\vec{\sigma}$ & $\hat{0}$ & & $\ddot{\circ}$ & $\stackrel{\delta}{0}$ & & $\stackrel{8}{\circ}$ & & $\begin{array}{l}\tilde{\sigma} \\
\tilde{\sigma} \\
\alpha\end{array}$ & & $\tilde{\delta}$ & $\begin{array}{l}\infty \\
\stackrel{\infty}{n} \\
i n\end{array}$ & $\begin{array}{l}\text { to } \\
\dot{0}\end{array}$ & $\begin{array}{l}\stackrel{\sigma}{\sigma} \\
\dot{\gamma}\end{array}$ & $\begin{array}{l}\stackrel{\sim}{i} \\
\text { }\end{array}$ & $\stackrel{0}{0}$ & $\stackrel{\delta}{0}$ & $\stackrel{t}{0}$ & $\overrightarrow{0}$ & & $\stackrel{\infty}{\circ}$ & $\begin{array}{l}\hat{\alpha} \\
\hat{\alpha}\end{array}$ \\
\hline $\bar{\lambda}$ & & $\stackrel{\infty}{\circ}$ & ?ొ & $\stackrel{n}{n}$ & $\stackrel{\infty}{+}$ & $\stackrel{m}{0}$ & & $\stackrel{0}{\circ}$ & $\stackrel{\text { ô }}{0}$ & & $\stackrel{7}{0}$ & & $\begin{array}{l}\stackrel{o}{+} \\
\text { a }\end{array}$ & & $\stackrel{0}{0}$ & $\begin{array}{l}\infty \\
\sim \\
\sim \\
n\end{array}$ & 志 & $\begin{array}{l}\partial \\
\dot{\gamma}\end{array}$ & त्र & $\stackrel{m}{0}$ & $\begin{array}{l}\tilde{\delta} \\
\text { Oे }\end{array}$ & $\stackrel{t}{\circ}$ & $\overrightarrow{\dot{0}}$ & & $\stackrel{\infty}{\circ}$ & à. \\
\hline సิ & & $\stackrel{8}{\circ}$ & $\bar{n}$ & $\stackrel{\text { ñ }}{0}$ & $\stackrel{m}{\vec{\alpha}}$ & ¿̊. & & $\stackrel{\leftrightarrow}{0}$ & $\stackrel{n}{0}$ & & $\stackrel{8}{\circ}$ & & $\begin{array}{l}\bar{z} \\
2\end{array}$ & & $\stackrel{n}{\circ}$ & 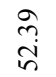 & $\stackrel{8}{\circ}$ & $\begin{array}{l}\stackrel{0}{+} \\
\dot{f}\end{array}$ & $\stackrel{\overbrace{}}{i}$ & $\stackrel{\widetilde{\sigma}}{\circ}$ & & & $\stackrel{\overbrace{}}{0}$ & $\stackrel{0}{0}$ & $\stackrel{\infty}{\circ}$ & $\tilde{m}$ \\
\hline 2 & & $\stackrel{\infty}{\circ}$ & fo & $\stackrel{\overbrace{}}{0}$ & $\begin{array}{c}\hat{\infty} \\
\stackrel{\alpha}{a}\end{array}$ & $\stackrel{\leftrightarrow}{0}$ & & $\overrightarrow{0}$ & $\stackrel{\infty}{0}$ & $\overline{0}$ & $\stackrel{\circ}{0}$ & & $\begin{array}{l}n \\
\infty \\
\alpha \\
\alpha\end{array}$ & & $\stackrel{0}{\circ}$ & $\frac{\vec{\sigma}}{\vec{n}}$ & & $\stackrel{\vartheta}{\dot{f}}$ & $\stackrel{\widetilde{i}}{i}$ & $\stackrel{n}{0}$ & $\stackrel{\sigma}{0}$ & $\ddot{\circ}$ & & $\stackrel{n}{0}$ & $\stackrel{\infty}{0}$ & $\begin{array}{l}\infty \\
\stackrel{2}{\alpha} \\
\alpha\end{array}$ \\
\hline$\stackrel{\infty}{=}$ & & $\stackrel{\circ}{0}$ & $\stackrel{0}{+}$ & 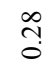 & $\begin{array}{l}\infty \\
\text { ¿̇ } \\
\alpha\end{array}$ & $\stackrel{m}{0}$ & & $\stackrel{0}{0}$ & $\stackrel{8}{\circ}$ & $\bar{\circ}$ & to & & $\frac{n}{a}$ & & $\ddot{\circ}$ & 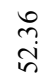 & $\overrightarrow{0}$ & \begin{tabular}{l}
$\tilde{n}$ \\
\multirow{z}{*}{}
\end{tabular} & $\stackrel{\infty}{\vec{i}}$ & $\stackrel{\Xi}{0}$ & $\stackrel{\circ}{\circ}$ & & & $\overrightarrow{0}$ & $\stackrel{\infty}{\circ}$ & $\stackrel{m}{\dot{8}}$ \\
\hline$=$ & & $\stackrel{\infty}{0}$ & ले & గ్ర & $\frac{\vec{n}}{a}$ & $\stackrel{8}{\circ}$ & & $\stackrel{0}{0}$ & $\stackrel{8}{\circ}$ & $\ddot{\circ}$ & $\stackrel{t}{0}$ & & 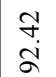 & & $\stackrel{n}{0}$ & $\begin{array}{l}\text { to } \\
\text { in }\end{array}$ & & $\begin{array}{l}\tilde{\sigma} \\
\dot{y}\end{array}$ & $\stackrel{\vec{o}}{i}$ & $\stackrel{8}{0}$ & $\stackrel{\circ}{\circ}$ & $\stackrel{\leftrightarrow}{\circ}$ & & $\stackrel{0}{0}$ & $\stackrel{\infty}{0}$ & $\begin{array}{l}\text { ते } \\
\dot{8}\end{array}$ \\
\hline $\mathscr{0}$ & & $\stackrel{7}{3}$ & $\stackrel{\infty}{\overbrace{0}^{0}}$ & $\stackrel{n}{0}$ & $\underset{⿱ \pi}{ \pm}$ & $\stackrel{t}{0}$ & $\overrightarrow{0}$ & $\stackrel{8}{0}$ & $\stackrel{\leftrightarrow}{0}$ & & $\stackrel{\circ}{\circ}$ & & 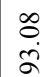 & & $\stackrel{\Delta}{0}$ & $\underset{i}{\mathbb{i}}$ & & $\begin{array}{l}n \\
\dot{f} \\
\dot{J}\end{array}$ & $\stackrel{\sim}{i}$ & ô. & $\vec{\circ}$ & $\stackrel{\leftrightarrow}{0}$ & $\stackrel{\Delta}{0}$ & & $\stackrel{\infty}{0}$ & ले \\
\hline 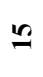 & & :े. & $\stackrel{n}{9}$ & $\stackrel{n}{0}$ & $\stackrel{\hat{a}}{\stackrel{a}{a}}$ & $\stackrel{t}{0}$ & & $\stackrel{\sigma}{0}$ & $\stackrel{\leftrightarrow}{\circ}$ & & $\stackrel{8}{0}$ & & $\begin{array}{c}: \\
\stackrel{0}{i} \\
\text { a }\end{array}$ & & $\stackrel{\overbrace{}}{\circ}$ & $\underset{\text { ì }}{\text { în }}$ & $\stackrel{\leftrightarrow}{\circ}$ & $\begin{array}{l}\bar{n} \\
\dot{+}\end{array}$ & $\stackrel{\sim}{\sim}$ & $\stackrel{\widetilde{\sigma}}{\circ}$ & $\overline{0}$ & & & $\overline{0}$ & $\stackrel{\infty}{\circ}$ & $\vec{m}$ \\
\hline$\Xi$ & & $\stackrel{0}{0}$ & $\underset{0}{J}$ & ô & $\stackrel{\beta}{a}$ & $\ddot{\circ}$ & & & $\stackrel{0}{0}$ & & $\stackrel{8}{\circ}$ & & $\begin{array}{l}\infty \\
n \\
i \\
i\end{array}$ & & $\stackrel{0}{0}$ & $\underset{i}{\stackrel{\forall}{i}}$ & $\overrightarrow{0}$ & \begin{tabular}{l}
$\stackrel{2}{y}$ \\
\multirow{J}{*}{}
\end{tabular} & $\stackrel{\infty}{\vec{i}}$ & $\overrightarrow{0}$ & $\stackrel{\delta}{0}$ & $\stackrel{\leftrightarrow}{0}$ & $\stackrel{\overbrace{}}{0}$ & $\stackrel{\text { ô }}{0}$ & $\stackrel{\infty}{\circ}$ & $\begin{array}{l}8 \\
\dot{0}\end{array}$ \\
\hline 9 & & $\stackrel{\circ}{\circ}$ & $\stackrel{f}{0}$ & $\begin{array}{l}\text { ก̊ } \\
0\end{array}$ & $\underset{\stackrel{\Xi}{L}}{ \pm}$ & oे & $\vec{\circ}$ & $\stackrel{8}{0}$ & $\stackrel{0}{\circ}$ & & $\stackrel{0}{0}$ & & $\frac{n}{m}$ & & $\ddot{0}$ & $\begin{array}{l}\tilde{N} \\
\stackrel{i}{i}\end{array}$ & $\overrightarrow{0}$ & $\begin{array}{l}\infty \\
\dot{+} \\
\dot{+}\end{array}$ & $\begin{array}{l}\tilde{O} \\
\text { i }\end{array}$ & $\stackrel{0}{0}$ & $\stackrel{\delta}{0}$ & $\stackrel{\sigma}{0}$ & & $\overrightarrow{0}$ & $\stackrel{\infty}{\circ}$ & $\stackrel{ \pm}{\dot{8}}$ \\
\hline$\approx$ & 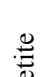 & $\stackrel{\circ}{0}$ & ?ై & రి & $\begin{array}{l}0 \\
\tilde{n} \\
\tilde{\alpha}\end{array}$ & $\stackrel{t}{\circ}$ & & $\stackrel{8}{0}$ & $\stackrel{n}{0}$ & & & & $\frac{\infty}{\cdots}$ & & $\ddot{\circ}$ & $\begin{array}{l}\underset{\sim}{1} \\
\underset{n}{n}\end{array}$ & $\overrightarrow{0}$ & $\begin{array}{l}\infty \\
\infty \\
\dot{J}\end{array}$ & $\stackrel{\text { Oे }}{\mathrm{i}}$ & $\stackrel{\leftrightarrow}{0}$ & $\stackrel{\sigma}{\circ}$ & ชั & & $\overline{0}$ & $\stackrel{\infty}{0}$ & $\frac{ \pm}{\dot{8}}$ \\
\hline$=$ & 超 & $\stackrel{\circ}{\circ}$ & $\stackrel{f}{\stackrel{f}{0}}$ & ते & $\frac{n}{a}$ & $\stackrel{\widetilde{\sigma}}{0}$ & & $\overrightarrow{0}$ & $\stackrel{\partial}{0}$ & & & & $\begin{array}{c}\stackrel{0}{ } \\
\text { a }\end{array}$ & $\stackrel{g}{g}$ & $\stackrel{\overbrace{}}{0}$ & $\begin{array}{l}\text { ర్ } \\
\text { i } \\
\text { in }\end{array}$ & $\begin{array}{l}\text { ô. } \\
\stackrel{0}{0}\end{array}$ & $\begin{array}{l}\stackrel{8}{2} \\
\stackrel{+}{+}\end{array}$ & $\stackrel{\widetilde{T}}{\mathrm{i}}$ & $\stackrel{\widetilde{O}}{0}$ & & 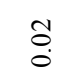 & $\stackrel{\text { ô. }}{\circ}$ & & $\stackrel{\infty}{\circ}$ & 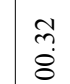 \\
\hline$\varrho$ & & $\stackrel{\circ}{\circ}$ & $\stackrel{n}{0}$ & $\bar{n}$ & $\begin{array}{l}\hat{b} \\
\text { ì }\end{array}$ & $\stackrel{t}{0}$ & & & 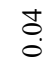 & & $\stackrel{n}{0}$ & & 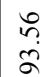 & & $\stackrel{m}{0}$ & 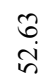 & $\vec{\circ}$ & $\begin{array}{l}\text { f } \\
\dot{f}\end{array}$ & $\underset{\text { î }}{\text { i }}$ & & $\vec{\circ}$ & $\tilde{O}$ & & $\stackrel{0}{0}$ & $\stackrel{\infty}{\circ}$ & $\begin{array}{l}\infty \\
\stackrel{\infty}{8} \\
\stackrel{8}{8}\end{array}$ \\
\hline$a$ & & $\stackrel{ \pm}{0}$ & $\stackrel{q}{9}$ & $\stackrel{n}{0}$ & $\begin{array}{l}\text { त్ } \\
\text { הू }\end{array}$ & $\stackrel{\infty}{\circ}$ & & & $\stackrel{7}{0}$ & & $\stackrel{8}{\circ}$ & & $\underset{\tilde{n}}{\tilde{\alpha}}$ & & & $\stackrel{\vec{i}}{\vec{i}}$ & & $\begin{array}{l}\vec{n} \\
\dot{y}\end{array}$ & $\overrightarrow{\vec{n}}$ & $\stackrel{8}{0}$ & $\tilde{O}$ & & $\stackrel{8}{\circ}$ & & $\stackrel{\infty}{0}$ & $\begin{array}{l}\mathscr{0} \\
\dot{8}\end{array}$ \\
\hline$\infty$ & & $\stackrel{5}{0}$ & $\underset{0}{F}$ & $\stackrel{m}{0}$ & $\stackrel{t}{\dot{a}}$ & $\stackrel{8}{\circ}$ & $\stackrel{t}{0}$ & $\overrightarrow{0}$ & $\stackrel{n}{0}$ & & $\stackrel{\wp}{0}$ & & ô. & & $\stackrel{1}{0}$ & $\begin{array}{l}8 \\
\text { i } \\
i\end{array}$ & & $\begin{array}{l}\mathscr{\infty} \\
\dot{J}\end{array}$ & $\stackrel{\infty}{\underset{\sim}{i}}$ & & $\stackrel{\sigma}{0}$ & & & $\begin{array}{l}\text { to } \\
0\end{array}$ & $\stackrel{\infty}{0}$ & $\begin{array}{l}\text { तิ } \\
\dot{8}\end{array}$ \\
\hline$r$ & & $=$ & $\hat{3}$ & 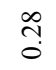 & 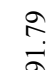 & $\stackrel{1}{0}$ & $\tilde{\delta}$ & $\overrightarrow{0}$ & $\hat{o}$ & & & & $\begin{array}{l}\hat{b} \\
i \\
i\end{array}$ & & & 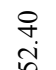 & $\stackrel{0}{0}$ & $\begin{array}{l}+ \\
+ \\
+\end{array}$ & $\stackrel{\infty}{\mathbb{N}}$ & & $\tilde{\delta}$ & $\stackrel{n}{0}$ & & & $\stackrel{\infty}{\circ}$ & $\begin{array}{l}\dot{0} \\
\dot{8}\end{array}$ \\
\hline 0 & & $\stackrel{0}{\circ}$ & $\begin{array}{l}n \\
0 \\
0\end{array}$ & స̃ & $\stackrel{\vec{i}}{i}$ & $\stackrel{0}{0}$ & & & $\stackrel{\infty}{0} 0$ & & & & $\bar{m}$ & & $\ddot{0}$ & $\begin{array}{l}\text { nิ } \\
\text { nn }\end{array}$ & 8 & $\begin{array}{l}\bar{m} \\
\dot{+}\end{array}$ & तે & $\overrightarrow{0}$ & $\stackrel{\delta}{0}$ & $\stackrel{\leftrightarrow}{0}$ & & $\stackrel{0}{0}$ & $\stackrel{\infty}{\circ}$ & $\begin{array}{l}\tilde{\imath} \\
\stackrel{8}{8}\end{array}$ \\
\hline in & & $\stackrel{\infty}{\circ}$ & 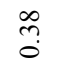 & $\stackrel{n}{n}$ & \begin{tabular}{l}
$\infty$ \\
\multirow{N}{N}{} \\
a
\end{tabular} & $\stackrel{\sigma}{0}$ & & $\stackrel{8}{\circ}$ & $\stackrel{o}{0}$ & $\stackrel{\dddot{o}}{0}$ & & & $\stackrel{m}{m}$ & & $\stackrel{8}{\circ}$ & $\underset{⿱ 亠}{\infty}$ & $\stackrel{t}{0}$ & 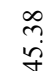 & $\stackrel{m}{i}$ & $\stackrel{0}{0}$ & $\stackrel{0}{0}$ & $\stackrel{\leftrightarrow}{0}$ & & $\stackrel{\tilde{O}}{0}$ & $\stackrel{\infty}{0}$ & $\begin{array}{l}0 \\
\text { ñ? } \\
8\end{array}$ \\
\hline+ & & $\exists$ & $\vec{n}$ & $\begin{array}{l}0 \\
0 \\
0\end{array}$ & $\begin{array}{l}8 \\
\text { i }\end{array}$ & $\exists$ & $\stackrel{t}{0}$ & $\stackrel{\text { ô. }}{0}$ & $\stackrel{n}{0}$ & $\stackrel{\leftrightarrow}{0}$ & $\stackrel{0}{\circ}$ & & $\vec{\sim}$ & & $\stackrel{0}{0}$ & $\stackrel{a}{\vec{n}}$ & $\overrightarrow{0}$ & $\begin{array}{l}\stackrel{\partial}{\infty} \\
\dot{+}\end{array}$ & $\stackrel{\vec{i}}{\vec{i}}$ & & $\tilde{\delta}$ & $\stackrel{0}{0}$ & & $\stackrel{m}{0}$ & $\stackrel{\infty}{\circ}$ & तે \\
\hline$m$ & & $\stackrel{\circ}{\circ}$ & in & $\stackrel{\infty}{\stackrel{\infty}{0}}$ & 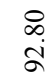 & $\overrightarrow{0}$ & o̊ & ô. & $\exists$ & & & & $\begin{array}{l}n \\
\alpha \\
\alpha\end{array}$ & & $\ddot{\circ}$ & $\underset{\sim}{=}$ & & $\begin{array}{l}\stackrel{\infty}{\infty} \\
\dot{+}\end{array}$ & $\frac{n}{i}$ & $\tilde{\delta}$ & ơ & $\stackrel{\tilde{s}}{\circ}$ & & $\stackrel{2}{\circ}$ & $\stackrel{\infty}{\circ}$ & $\begin{array}{l}\text { mे } \\
\stackrel{8}{8}\end{array}$ \\
\hline$N$ & & $\stackrel{\infty}{0}$ & $\stackrel{\substack{0 \\
0}}{0}$ & $\overrightarrow{\widetilde{o}}$ & $\underset{\Delta}{\Delta}$ & $\stackrel{\dddot{0}}{\circ}$ & $\stackrel{t}{0}$ & $\stackrel{\sigma}{0}$ & $\stackrel{0}{0}$ & & $\stackrel{\circ}{\circ}$ & & $\begin{array}{c}\bar{n} \\
\tilde{n}\end{array}$ & & 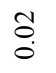 & $\begin{array}{l}\infty \\
i \\
i \\
i n\end{array}$ & & $\begin{array}{l}\dot{J} \\
\dot{J}\end{array}$ & $\stackrel{\vec{i}}{i}$ & & $\ddot{\circ}$ & $\stackrel{0}{0}$ & $\ddot{\circ}$ & & $\stackrel{\infty}{\circ}$ & $\begin{array}{l}\text { aे } \\
\text { aे }\end{array}$ \\
\hline- & & $\stackrel{\leftrightarrow}{0}$ & $\hat{n}$ & 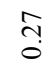 & $\begin{array}{l}\stackrel{+}{+} \\
\text { a }\end{array}$ & $\stackrel{\sigma}{\circ}$ & & $\stackrel{\sigma}{0}$ & $\stackrel{m}{\circ}$ & & & & $\underset{m}{m}$ & & & $\underset{\vec{i}}{\vec{i}}$ & & $\begin{array}{l}\infty \\
\stackrel{0}{+} \\
\dot{f}\end{array}$ & $\underset{\substack{i \\
i}}{ }$ & $\stackrel{8}{0}$ & $\stackrel{\sigma}{\circ}$ & ชั & & ó. & $\stackrel{\infty}{0}$ & $\frac{\infty}{\dot{8}}$ \\
\hline ज & $\dot{\Xi}$ & $\stackrel{\circ}{\infty}$ & $\stackrel{\Omega^{N}}{\Omega}$ & $\stackrel{0}{\frac{0}{2}}$ & O & $\stackrel{\wp}{E}$ & $\sum_{\sum}^{\infty}$ & ర్ & $\begin{array}{l}0 \\
0 \\
-1\end{array}$ & $\stackrel{\rho}{\mathrm{z}}$ & 용 & & 产 & $\dot{\Xi}$ & $\stackrel{\circ}{\infty}$ & $\stackrel{\overbrace{}^{N}}{:}$ & $\stackrel{O^{\infty}}{\gtrless}$ & o & $\stackrel{\wp}{E}$ & $\sum_{\sum}^{\infty}$ & ర్ల & $\begin{array}{l}0 \\
0 \\
0\end{array}$ & $\stackrel{\circ}{\stackrel{\circ}{Z}}$ & $\stackrel{O}{\mathcal{N}}$ & $0^{m}$ & 吾 \\
\hline
\end{tabular}




\begin{tabular}{|c|c|c|c|c|c|c|c|c|c|c|c|c|c|c|c|c|c|c|c|c|c|c|c|c|c|c|}
\hline$\exists$ & & $\stackrel{\circ}{\circ}$ & 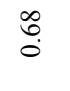 & $\stackrel{\text { ஸे }}{0}$ & $\frac{a}{\dot{a}}$ & $\stackrel{\infty}{\stackrel{0}{0}}$ & $\stackrel{8}{\circ}$ & $\stackrel{8}{0}$ & & ठे. & & สุ & 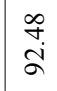 & & $\stackrel{\delta}{\delta}$ & $\begin{array}{l}\text { ñ } \\
\text { in }\end{array}$ & $\stackrel{8}{8}$ & $\begin{array}{l}\infty \\
\dot{f} \\
\dot{f}\end{array}$ & $\stackrel{i}{i}$ & $\stackrel{\widetilde{\sigma}}{\circ}$ & $\stackrel{0}{\circ}$ & $\overline{0}$ & & & $\underset{\text { a }}{\text {. }}$ & $\stackrel{n}{a}$ \\
\hline$\vartheta$ & & $\stackrel{?}{\circ}$ & ?̊ & 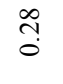 & $\begin{array}{l}\dot{D} \\
\dot{a}\end{array}$ & $\stackrel{\circ}{\circ}$ & $\stackrel{\circ}{\circ}$ & $\begin{array}{l}\widetilde{O} \\
\text { o. }\end{array}$ & & ס̊. & & สิ & $\begin{array}{l}\hat{\sigma} \\
\text { d. }\end{array}$ & & $\stackrel{8}{\circ}$ & $\stackrel{n}{\stackrel{n}{n}}$ & $\stackrel{0}{0}$ & $\hat{\alpha}$ & $\overrightarrow{\mathrm{N}}$ & Oे. & రั & $\stackrel{8}{\circ}$ & & & $\stackrel{\infty}{\stackrel{\infty}{\circ}}$ & o. \\
\hline F & & $\stackrel{\Delta}{0}$ & ñ & $\stackrel{\text { ñ }}{0}$ & $\stackrel{\grave{m}}{\vec{a}}$ & $\stackrel{1}{0}$ & 8 & $\stackrel{0}{\circ}$ & & o̊ & & $\frac{9}{0}$ & $\begin{array}{l}\infty \\
n \\
\tilde{a} \\
\alpha\end{array}$ & & $\overrightarrow{0}$ & $\begin{array}{l}\stackrel{n}{0} \\
\text { in }\end{array}$ & $\ddot{\circ}$ & $\begin{array}{l}\mathbb{J} \\
\dot{J}\end{array}$ & $\stackrel{n}{\text { ch }}$ & $\stackrel{\overbrace{}}{0}$ & $\ddot{\circ}$ & 8 & & & $\stackrel{\circ}{=}$ & $\begin{array}{l}a \\
\infty \\
a\end{array}$ \\
\hline$F$ & & $\stackrel{0}{\circ}$ & ț & 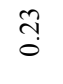 & $\stackrel{n}{\stackrel{n}{\sigma}}$ & $\stackrel{\circ}{0}$ & $\stackrel{8}{0}$ & $\stackrel{\square}{\circ}$ & & $\begin{array}{l}\stackrel{0}{\circ} \\
0 \\
0\end{array}$ & & $\exists$ & $\begin{array}{l}8 \\
\dot{+}\end{array}$ & & $\vec{a}$ & $\underset{\dot{b}}{\dot{m}}$ & $\stackrel{1}{0}$ & $\begin{array}{l}\stackrel{n}{q} \\
\dot{f}\end{array}$ & $\stackrel{\infty}{-}$ & $\stackrel{0}{0}$ & $\stackrel{8}{\circ}$ & $\stackrel{\text { Oे }}{0}$ & & & $\stackrel{\infty}{\stackrel{0}{0}}$ & $\begin{array}{l}0 \\
0 \\
\infty \\
\alpha\end{array}$ \\
\hline \& & & $\stackrel{t}{0}$ & $\begin{array}{l}3 \\
0 \\
0\end{array}$ & $\vec{ก}$ & $\frac{n}{\pi}$ & $\stackrel{8}{\circ}$ & $\vec{\circ}$ & $\stackrel{\Xi}{\circ}$ & & $\stackrel{0}{\circ}$ & & $\vec{\jmath}$ & $\begin{array}{l}\hat{i} \\
\text { a }\end{array}$ & & $\stackrel{8}{0}$ & $\underset{\substack{i \\
i n}}{ }$ & $\stackrel{1}{0}$ & $\begin{array}{l}\tilde{Y} \\
\dot{J}\end{array}$ & $\underset{i}{\stackrel{\infty}{i}}$ & $\stackrel{8}{0}$ & $\ddot{0}$ & $\stackrel{8}{0}$ & & & $\stackrel{n}{=}$ & 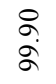 \\
\hline ले & & $\stackrel{\infty}{\circ}$ & $\stackrel{n}{s}$ & $\stackrel{n}{0}$ & $\begin{array}{l}\hat{\sigma} \\
\dot{\sigma}\end{array}$ & $\overline{0}$ & $\stackrel{\text { ô. }}{0}$ & $\stackrel{\circ}{\circ}$ & & $\stackrel{n}{\circ}$ & & ঙิ & $\begin{array}{l}\dot{0} \\
\dot{\sigma}\end{array}$ & & $\overrightarrow{0}$ & $\stackrel{\infty}{\stackrel{\infty}{n}}$ & $\tilde{\delta}$ & $\hat{\tilde{n}}$ & $\stackrel{\infty}{\underset{i}{i}}$ & $\stackrel{8}{0}$ & $\ddot{\circ}$ & $\stackrel{\Omega}{0}$ & & & $\underset{\overbrace{}}{\widetilde{C}}$ & $=$ \\
\hline$\infty$ & & $\stackrel{\overbrace{}}{0}$ & $\ddot{b}$ & ֶֻ & $\begin{array}{l}\dot{t} \\
\dot{a}\end{array}$ & 8 & $\ddot{\circ}$ & $\stackrel{8}{\circ}$ & & o. & & $\stackrel{\circ}{\circ}$ & $\begin{array}{l}\underset{\text { J }}{\text { S }} \\
\text {. }\end{array}$ & & $\overrightarrow{0}$ & $\underset{\stackrel{\infty}{\uparrow}}{\stackrel{n}{n}}$ & ฮ̊. & $\hat{\tilde{n}}$ & $\stackrel{\infty}{i}$ & $\stackrel{8}{0}$ & $\ddot{\circ}$ & $\stackrel{\mathrm{s}}{\mathrm{O}}$ & & & $\underset{\sim}{\sigma}$ & $=$ \\
\hline so & & $\stackrel{8}{\circ}$ & in & $\stackrel{\text { ñ }}{0}$ & 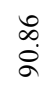 & ó. & 8 & $\stackrel{\text { ô. }}{\circ}$ & & $\stackrel{n}{\circ}$ & & $\stackrel{5}{0}$ & $\begin{array}{c}\hat{a} \\
\hat{a}\end{array}$ & & $\stackrel{t}{0}$ & 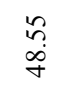 & $\stackrel{8}{\circ}$ & $\begin{array}{l}\stackrel{g}{q} \\
\dot{f}\end{array}$ & $\underset{i}{i}$ & 8 & $\ddot{\circ}$ & $\stackrel{\overbrace{}}{\circ}$ & & & $\stackrel{\infty}{\circ}$ & $\begin{array}{l}n \\
n \\
\infty \\
\infty\end{array}$ \\
\hline ల & & $\stackrel{\circ}{\circ}$ & $\begin{array}{l}\infty \\
\infty \\
0\end{array}$ & 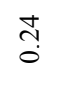 & 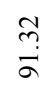 & $\overrightarrow{0}$ & $\stackrel{8}{\circ}$ & $\stackrel{8}{\circ}$ & & ¿̊. & & $\stackrel{n}{\circ}$ & $\begin{array}{l}n \\
i \\
i \\
\sigma\end{array}$ & & $\overrightarrow{0}$ & $\begin{array}{l}\text { fa } \\
\stackrel{8}{8}\end{array}$ & $\stackrel{\square}{\circ}$ & 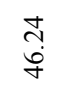 & $\overrightarrow{\tilde{n}}$ & $\ddot{\circ}$ & $\stackrel{8}{0}$ & $\stackrel{0}{\circ}$ & & & $\underset{-}{\stackrel{t}{0}}$ & $\begin{array}{l}\dot{0} \\
\dot{0}\end{array}$ \\
\hline$\ddot{m}$ & & $\stackrel{\Delta}{0}$ & $\stackrel{8}{0}$ & $\stackrel{\text { กิ }}{0}$ & $\stackrel{m}{\dot{q}}$ & $\stackrel{\dddot{0}}{0}$ & 8 & $\stackrel{\text { ô. }}{\circ}$ & & 8 & & $\frac{9}{0}$ & $\begin{array}{l}\dot{\$} \\
\stackrel{\infty}{\sigma}\end{array}$ & & $\overrightarrow{0}$ & $\stackrel{m}{\vec{n}}$ & $\stackrel{\square}{\circ}$ & $\hat{\sigma}$ & $\underset{\mathrm{i}}{\mathrm{i}}$ & 8 & $\ddot{\circ}$ & $\stackrel{8}{\circ}$ & & & $\stackrel{ }{=}$ & $\begin{array}{l}\stackrel{2}{a} \\
\infty \\
\infty\end{array}$ \\
\hline$\vec{m}$ & $\stackrel{\Xi}{\mathscr{E}}$ & $\stackrel{0}{0}$ & $\stackrel{8}{:}$ & $\stackrel{7}{0}$ & बे. & $\stackrel{\infty}{0}$ & $\vec{\circ}$ & ¿. & & $\frac{9}{\circ}$ & & $\stackrel{5}{0}$ & $\begin{array}{l}\stackrel{m}{i} \\
\text { a }\end{array}$ & 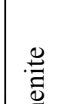 & $\stackrel{\check{\rho}}{\circ}$ & $\begin{array}{l}\vec{\sigma} \\
\stackrel{i}{n}\end{array}$ & $\stackrel{0}{0}$ & 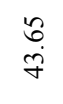 & $\underset{\mathrm{i}}{\mathrm{i}}$ & $\overrightarrow{0}$ & $\ddot{\circ}$ & $\stackrel{8}{\circ}$ & & & $\stackrel{n}{=}$ & $\hat{n}$ \\
\hline$m$ & $\begin{array}{l}E_{0} \\
\overparen{\Xi}\end{array}$ & 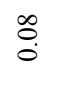 & $\begin{array}{l}\stackrel{0}{0} \\
\stackrel{0}{0}\end{array}$ & 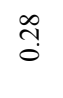 & $\stackrel{n}{a}$ & $\overrightarrow{0}$ & $\stackrel{8}{0}$ & ¿. & & $\overrightarrow{\widehat{N}}$ & & $\stackrel{\text { तి }}{0}$ & $\begin{array}{l}\infty \\
n \\
\\
\end{array}$ & $\stackrel{\Xi}{\Xi}$ & $\stackrel{8}{0}$ & $\begin{array}{l}\text { o } \\
\text { i } \\
\text { n. }\end{array}$ & $\stackrel{8}{0}$ & $\begin{array}{l}\infty \\
\stackrel{n}{p} \\
\stackrel{\gamma}{2}\end{array}$ & 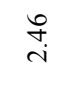 & $\stackrel{\tilde{o}}{\circ}$ & $\stackrel{8}{\circ}$ & $\ddot{0}$ & & & $\exists$ & $\begin{array}{l}\hat{n} \\
\alpha\end{array}$ \\
\hline$\tilde{\tilde{n}}$ & & $\stackrel{\Delta}{\circ}$ & 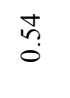 & $\stackrel{\tilde{\pi}}{0}$ & $\underset{\stackrel{+}{a}}{\sigma}$ & $\stackrel{\infty}{0}$ & $\stackrel{8}{0}$ & & & $\begin{array}{l}0 \\
0 \\
0\end{array}$ & & & $\begin{array}{l}\text { ते } \\
\text { ૂू }\end{array}$ & & $\stackrel{0}{0}$ & 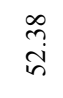 & $\stackrel{8}{\circ}$ & $\begin{array}{l}\overline{\mathrm{j}} \\
\vec{y}\end{array}$ & $\stackrel{+}{\stackrel{+}{i}}$ & $\overrightarrow{0}$ & $\overrightarrow{0}$ & $\stackrel{8}{\circ}$ & & & $\underset{\sim}{\stackrel{\sim}{Z}}$ & $\begin{array}{l}\infty \\
\infty \\
\infty \\
\infty\end{array}$ \\
\hline $\bar{m}$ & & $\stackrel{t}{0}$ & $\begin{array}{l}+ \\
\infty \\
0\end{array}$ & तิ & $\underset{\square}{ \pm}$ & ô. & $\stackrel{0}{\circ}$ & & & $\frac{n}{0}$ & & & 年 & & 8 & $\underset{\substack{n \\
i}}{i}$ & $\stackrel{8}{0}$ & $\begin{array}{l}\bar{g} \\
\vec{y}\end{array}$ & $\stackrel{\circ}{\stackrel{0}{i}}$ & $\vec{\circ}$ & $\stackrel{\square}{\circ}$ & $\stackrel{8}{\circ}$ & & & $\stackrel{\overbrace{}}{\beth}$ & $\begin{array}{l}\infty \\
\infty \\
\infty \\
\infty\end{array}$ \\
\hline లి & & $\stackrel{\circ}{\circ}$ & ले & $\stackrel{\text { ஸ̣ }}{0}$ & $\stackrel{\infty}{\stackrel{n}{a}}$ & $\stackrel{d}{0}$ & & $\stackrel{8}{\circ}$ & & $\stackrel{0}{\circ}$ & & & 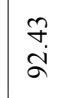 & & & $\underset{i}{i}$ & $\stackrel{\Omega}{\circ}$ & $\begin{array}{l}\stackrel{+}{\infty} \\
\dot{f}\end{array}$ & $\begin{array}{l}\vec{b} \\
\vec{i}\end{array}$ & $\underset{\delta}{0}$ & $\tilde{O}$ & $\stackrel{\leftrightarrow}{0}$ & & $\overrightarrow{0}$ & $\stackrel{\infty}{\stackrel{0}{0}}$ & 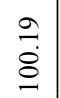 \\
\hline ते & & $\stackrel{8}{\circ}$ & fọ & $\stackrel{\text { ָ̦ }}{0}$ & $\stackrel{\circ}{\stackrel{\circ}{a}}$ & $\stackrel{0}{0}$ & & $\stackrel{\sim}{\sigma}$ & & $\stackrel{8}{\stackrel{2}{0}}$ & $\stackrel{\leftrightarrow}{\circ}$ & & $\begin{array}{l}\infty \\
\\
\tilde{\alpha}\end{array}$ & & & $\begin{array}{c}\hat{i} \\
i\end{array}$ & $\tilde{O}$ & $\begin{array}{l}+ \\
\infty \\
\dot{f}\end{array}$ & $\overrightarrow{\vec{i}}$ & $\stackrel{\widetilde{c}}{\circ}$ & 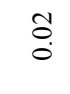 & $\stackrel{\overbrace{}}{0}$ & & $\overrightarrow{0}$ & $\stackrel{\infty}{\circ}$ & $\begin{array}{l}\stackrel{2}{\dot{8}} \\
\dot{8}\end{array}$ \\
\hline$\stackrel{\sim}{\sim}$ & & $\stackrel{\infty}{\circ}$ & $\underset{0}{\overrightarrow{0}}$ & $\vec{m}$ & $\stackrel{?}{\dot{a}}$ & $\stackrel{n}{0}$ & $\vec{\circ}$ & $\stackrel{\sigma}{\circ}$ & & ț & & & $\begin{array}{l}\hat{\sigma} \\
\dot{\alpha} \\
\alpha\end{array}$ & & $\stackrel{t}{0}$ & $\underset{\mathrm{i}}{\stackrel{+}{i}}$ & $\overrightarrow{0}$ & $\begin{array}{l}\stackrel{+}{n} \\
\stackrel{+}{+}\end{array}$ & $\underset{i}{\stackrel{d}{i}}$ & & $\stackrel{t}{0}$ & & $\stackrel{d}{0}$ & & $\stackrel{\infty}{\circ}$ & $\begin{array}{l}\infty \\
\stackrel{2}{\alpha} \\
\alpha\end{array}$ \\
\hline$\hat{N}$ & & $=$ & $\stackrel{t}{n}$ & $\stackrel{\text { ते }}{0}$ & $\stackrel{\stackrel{i}{i}}{a}$ & $\hat{0}$ & & : & & : & $\ddot{b}$ & & $\begin{array}{l}\hat{\infty} \\
\text { ja }\end{array}$ & & 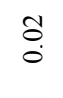 & $\begin{array}{l}n \\
\stackrel{n}{i}\end{array}$ & $\vec{\circ}$ & $\begin{array}{l}\stackrel{\infty}{\circ} \\
\stackrel{+}{+}\end{array}$ & $\stackrel{n}{a}$ & $\stackrel{n}{0}$ & ơ & $\stackrel{t}{0}$ & $\overrightarrow{0}$ & $\stackrel{n}{0}$ & $\stackrel{\infty}{\circ}$ & \begin{tabular}{l}
0 \\
$\tilde{\Xi}$ \\
$\stackrel{0}{0}$ \\
\hdashline
\end{tabular} \\
\hline$\stackrel{\sim}{\sim}$ & & $\stackrel{\circ}{\circ}$ & ?̊n & त̃ & $\stackrel{\text { f }}{-}$ & $\stackrel{\tilde{O}}{0}$ & & $\stackrel{\text { o. }}{0}$ & & $\frac{0}{0}$ & $\stackrel{n}{0}$ & & $\begin{array}{l}\text { in } \\
\text { in } \\
\text { a }\end{array}$ & & 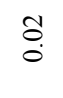 & $\stackrel{n}{i}$ & $\overrightarrow{0}$ & $\begin{array}{l}\infty \\
\stackrel{0}{f} \\
\dot{f}\end{array}$ & $\stackrel{n}{g}$ & $\stackrel{n}{0}$ & $\stackrel{\widetilde{O}}{0}$ & $\stackrel{\Delta}{0}$ & $\overrightarrow{0}$ & $\frac{n}{0}$ & $\stackrel{\infty}{\circ}$ & $\begin{array}{l}0 \\
\tilde{c} \\
\stackrel{8}{0} \\
\stackrel{0}{2}\end{array}$ \\
\hline$\stackrel{2}{\sim}$ & & $\stackrel{\circ}{\circ}$ & 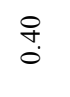 & ల్ల & $\stackrel{+}{\stackrel{+}{\sigma}}$ & ô. & & $\stackrel{\circ}{\circ}$ & & ¿े. & & & $\stackrel{n}{\tilde{j}}$ & & $\stackrel{\text { ô. }}{\circ}$ & $\begin{array}{l}\infty \\
i n \\
i n\end{array}$ & $\stackrel{8}{0}$ & 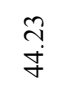 & $\stackrel{m}{\vec{i}}$ & $\stackrel{t}{0}$ & $\stackrel{\square}{\circ}$ & & $\overrightarrow{0}$ & $\stackrel{\Delta}{\circ}$ & $\stackrel{\infty}{\circ}$ & $\begin{array}{l}\tilde{o} \\
\dot{8} \\
0\end{array}$ \\
\hline$\vec{N}$ & & $\stackrel{\circ}{\circ}$ & 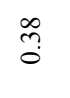 & $\stackrel{\hat{n}}{0}$ & aे. & o̊. & & $\sigma_{0}$ & & مُ & $\stackrel{\sigma}{0}$ & & $\begin{array}{l}\tilde{n} \\
\tilde{z}\end{array}$ & & $\overrightarrow{0}$ & $\begin{array}{l}\text { ț } \\
\text { in }\end{array}$ & $\stackrel{\text { Oे }}{\circ}$ & $\stackrel{\infty}{\underset{f}{J}}$ & $\stackrel{\infty}{\stackrel{N}{N}}$ & $\stackrel{\leftrightarrow}{0}$ & & & $\stackrel{8}{0}$ & $\stackrel{n}{\circ}$ & $\stackrel{\infty}{\circ}$ & 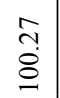 \\
\hline$\approx$ & & $\ddot{\leftrightarrow}$ & $\overrightarrow{\breve{0}}$ & $\tilde{\widetilde{Z}}$ & $\stackrel{?}{\stackrel{?}{\sigma}}$ & $\begin{array}{l}0 \\
0 \\
0\end{array}$ & & 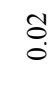 & & $\stackrel{n}{\circ}$ & $\stackrel{\circ}{\circ}$ & & 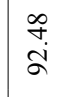 & & $\stackrel{\leftrightarrow}{\circ}$ & $\begin{array}{l}\infty \\
\text { ñ } \\
i \\
i\end{array}$ & $\stackrel{t}{0}$ & $\begin{array}{l}\text { बे } \\
\dot{\gamma}\end{array}$ & 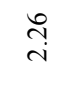 & $\stackrel{\tilde{o}}{0}$ & $\stackrel{\widetilde{c}}{\circ}$ & $\stackrel{\Delta}{0}$ & $\overrightarrow{0}$ & & $\stackrel{\infty}{\circ}$ & $\begin{array}{l}\hat{\alpha} \\
\hat{\alpha}\end{array}$ \\
\hline $\begin{array}{l}\dot{\dot{z}} \\
\dot{\bar{E}}\end{array}$ & $\dot{\dot{\Xi}}$ & $\stackrel{\rho}{n}^{N}$ & $\stackrel{\stackrel{O}{N}^{N}}{ }$ & $0^{m}$ & ) & $\stackrel{0}{E}$ & & & & 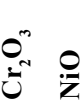 & 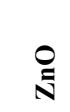 & $\begin{array}{l}0^{m} \\
\lambda^{n}\end{array}$ & 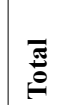 & 音 & $\stackrel{\rho^{n}}{n}$ & $\stackrel{\Omega}{*}^{\sim}$ & $\frac{0^{n}}{2}$ & $\stackrel{0}{0}$ & $\stackrel{\wp}{E}$ & $\begin{array}{l}\infty \\
\sum_{\Sigma}^{\infty}\end{array}$ & ర్ల & $\dot{0}^{m}$ & $\frac{0}{2}$ & 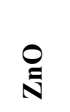 & $\begin{array}{c}0^{n} \\
>^{-1}\end{array}$ & 吾 \\
\hline
\end{tabular}



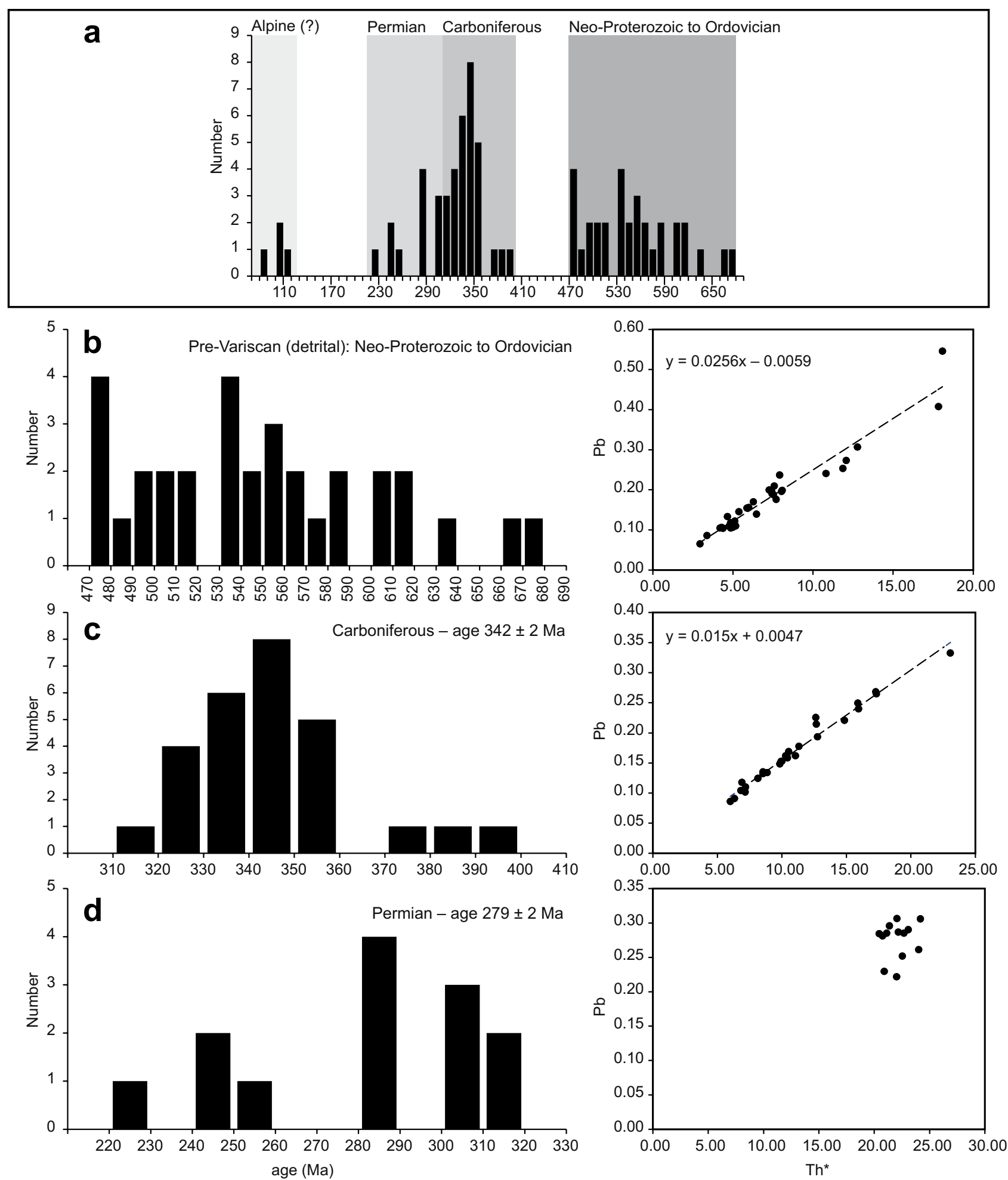

Fig. 4 Results of EPMA monazite dating. In the main histogram (a), the monazites fall into four principal groups: (i) monazites of detrital origin from the Neo-Proterozoic to Ordovician (b), (ii) Carboniferous monazites of metamorphic origin with a distinct maximum at c. 345 Ma (c), (iii) Permian monazites (d) and (iv) a few Alpine ages from monazites and one Th-U-Si phase (not plotted).

Variability in composition allows constructing isochrons for pre-Variscan and Carboniferous monazites (b-c). The fact that the isochrons pass through the origin provides an additional proof of the monazite dating reliability. Isochron cannot be fitted for Permian monazites with a too narrow compositional range, though $(\mathbf{d})$. The Th* value includes Th plus equivalent of U. Analyses are presented in Tabs 6-7. 
Tab. 5 Composition of coexisting Fe-Ti oxides given in the molar proportions of end members

\begin{tabular}{|c|c|c|c|c|c|c|c|c|c|c|c|}
\hline \multirow[b]{2}{*}{ Pair No. } & \multicolumn{3}{|c|}{ rhomboedral oxide } & \multicolumn{6}{|c|}{ cubic oxide } & \multirow[b]{2}{*}{$\begin{array}{c}\text { Temperature* } \\
\mathrm{T}\left({ }^{\circ} \mathrm{C}\right) 2 \mathrm{~s}\end{array}$} & \multirow[b]{2}{*}{$\begin{array}{c}\text { Oxygen } \\
\text { fugacity } \\
\mathrm{fO}_{2}\end{array}$} \\
\hline & $\begin{array}{c}\text { Hematite } \\
\text { XHem }\end{array}$ & $\begin{array}{c}\text { Geikielite } \\
\quad X G k\end{array}$ & $\begin{array}{c}\text { Pyrophanite } \\
X P y\end{array}$ & $\begin{array}{c}\text { Galaxite } \\
\text { XGal }\end{array}$ & $\begin{array}{c}\text { Ulvöspinel } \\
\qquad X U s p\end{array}$ & $\begin{array}{c}\text { Hercynite } \\
\text { XHer }\end{array}$ & $\begin{array}{c}\text { Magnesioferrite } \\
\qquad X M g\end{array}$ & $\begin{array}{c}\text { Magnetite } \\
\text { XMt }\end{array}$ & $\begin{array}{c}\text { Chromite } \\
\text { XChr }\end{array}$ & & \\
\hline 1 & 0.0121 & 0.0000 & 0.0491 & 0.0008 & 0.0164 & 0.0053 & 0.0000 & 0.9847 & 0.0005 & $371 \pm 66$ & -30.6 \\
\hline 2 & 0.0007 & 0.0000 & 0.0468 & 0.0009 & 0.0235 & 0.0038 & 0.0024 & 0.9786 & 0.0011 & $252 \pm 39$ & -49.2 \\
\hline 3 & 0.0130 & 0.0008 & 0.0460 & 0.0036 & 0.0144 & 0.0026 & 0.0020 & 0.9813 & 0.0017 & $375 \pm 20$ & -30.2 \\
\hline 4 & 0.0143 & 0.0000 & 0.0468 & 0.0034 & 0.0147 & 0.0034 & 0.0024 & 0.9805 & 0.0008 & $381 \pm 22$ & -29.6 \\
\hline 5 & 0.0197 & 0.0009 & 0.0431 & 0.0007 & 0.0110 & 0.0049 & 0.0000 & 0.9860 & 0.0013 & $377 \pm 66$ & -28.1 \\
\hline 6 & 0.0027 & 0.0005 & 0.0470 & 0.0024 & 0.0188 & 0.0026 & 0.0000 & 0.9826 & 0.0012 & $304 \pm 24$ & -40.3 \\
\hline 7 & 0.0113 & 0.0000 & 0.0484 & 0.0015 & 0.0108 & 0.0047 & 0.0009 & 0.9843 & 0.0010 & $350 \pm 41$ & -31.6 \\
\hline 8 & 0.0134 & 0.0000 & 0.0487 & 0.0021 & 0.0119 & 0.0055 & 0.0021 & 0.9805 & 0.0007 & $365 \pm 36$ & -30.3 \\
\hline 9 & 0.0095 & 0.0000 & 0.0494 & 0.0025 & 0.0115 & 0.0031 & 0.0000 & 0.9843 & 0.0018 & $345 \pm 27$ & -32.6 \\
\hline 10 & 0.0033 & 0.0000 & 0.0469 & 0.0012 & 0.0100 & 0.0058 & 0.0000 & 0.9858 & 0.0005 & $287 \pm 36$ & -39.9 \\
\hline 11 & 0.0034 & 0.0008 & 0.0453 & 0.0008 & 0.0127 & 0.0058 & 0.0000 & 0.9839 & 0.0013 & $295 \pm 49$ & -39.4 \\
\hline 12 & 0.0094 & 0.0005 & 0.0512 & 0.0012 & 0.0105 & 0.0056 & 0.0000 & 0.9859 & 0.0008 & $338 \pm 42$ & -32.8 \\
\hline 13 & 0.0094 & 0.0011 & 0.0431 & 0.0029 & 0.0135 & 0.0029 & 0.0004 & 0.9842 & 0.0009 & $353 \pm 21$ & -32.4 \\
\hline 14 & 0.0033 & 0.0005 & 0.0467 & 0.0004 & 0.0128 & 0.0065 & 0.0000 & 0.9843 & 0.0010 & $292 \pm 62$ & -39.7 \\
\hline 15 & 0.0063 & 0.0007 & 0.0480 & 0.0013 & 0.0130 & 0.0043 & 0.0000 & 0.9856 & 0.0005 & $328 \pm 42$ & -35.2 \\
\hline 16 & 0.0064 & 0.0009 & 0.0451 & 0.0013 & 0.0111 & 0.0044 & 0.0006 & 0.9853 & 0.0004 & $322 \pm 39$ & -35.3 \\
\hline 17 & 0.0132 & 0.0002 & 0.0443 & 0.0019 & 0.0113 & 0.0041 & 0.0000 & 0.9860 & 0.0009 & $361 \pm 34$ & -30.5 \\
\hline 18 & 0.0073 & 0.0014 & 0.0466 & 0.0044 & 0.0134 & 0.0020 & 0.0000 & 0.9847 & 0.0009 & $340 \pm 12$ & -34.1 \\
\hline 19 & 0.0133 & 0.0018 & 0.0453 & 0.0015 & 0.0138 & 0.0038 & 0.0000 & 0.9851 & 0.0012 & $371 \pm 44$ & -30.2 \\
\hline 20 & 0.0066 & 0.0009 & 0.0433 & 0.0008 & 0.0148 & 0.0049 & 0.0000 & 0.9851 & 0.0008 & $333 \pm 57$ & -34.8 \\
\hline 21 & 0.0007 & 0.0009 & 0.0483 & 0.0011 & 0.0104 & 0.0047 & 0.0000 & 0.9872 & 0.0002 & $228 \pm 30$ & -50.2 \\
\hline 22 & 0.0007 & 0.0009 & 0.0483 & 0.0022 & 0.0149 & 0.0039 & 0.0000 & 0.9840 & 0.0003 & $338 \pm 28$ & -34.6 \\
\hline 23 & 0.0007 & 0.0009 & 0.0483 & 0.0014 & 0.0178 & 0.0036 & 0.0000 & 0.9843 & 0.0008 & $345 \pm 41$ & -34.4 \\
\hline 24 & 0.0026 & 0.0006 & 0.0508 & 0.0024 & 0.0110 & 0.0037 & 0.0000 & 0.9862 & 0.0010 & $300 \pm 25$ & -40.1 \\
\hline 25 & 0.0012 & 0.0016 & 0.0455 & 0.0008 & 0.0115 & 0.0061 & 0.0000 & 0.9860 & 0.0002 & $353 \pm 60$ & -31.1 \\
\hline 26 & 0.0121 & 0.0019 & 0.0417 & 0.0005 & 0.0164 & 0.0044 & 0.0000 & 0.9833 & 0.0016 & $368 \pm 74$ & -30.7 \\
\hline 27 & 0.0121 & 0.0019 & 0.0417 & 0.0024 & 0.0156 & 0.0034 & 0.0000 & 0.9833 & 0.0010 & $366 \pm 74$ & -30.8 \\
\hline 28 & 0.0052 & 0.0000 & 0.0437 & 0.0016 & 0.0120 & 0.0054 & 0.0003 & 0.9844 & 0.0006 & $315 \pm 32$ & -36.7 \\
\hline 29 & 0.0096 & 0.0006 & 0.0429 & 0.0029 & 0.0117 & 0.0031 & 0.0000 & 0.9859 & 0.0005 & $347 \pm 20$ & -32.5 \\
\hline 30 & 0.0096 & 0.0006 & 0.0429 & 0.0013 & 0.0113 & 0.0045 & 0.0000 & 0.9855 & 0.0010 & $343 \pm 41$ & -32.6 \\
\hline 31 & 0.0059 & 0.0005 & 0.0531 & 0.0022 & 0.0244 & 0.0028 & 0.0000 & 0.9798 & 0.0023 & $355 \pm 33$ & -34.7 \\
\hline 32 & 0.0059 & 0.0005 & 0.0531 & 0.0026 & 0.0156 & 0.0027 & 0.0000 & 0.9836 & 0.0025 & $335 \pm 28$ & -35.3 \\
\hline 33 & 0.0007 & 0.0009 & 0.0528 & 0.0003 & 0.0221 & 0.0061 & 0.0002 & 0.9776 & 0.0031 & $338 \pm 89$ & -35.2 \\
\hline 34 & 0.0059 & 0.0005 & 0.0531 & 0.0026 & 0.0192 & 0.0012 & 0.0006 & 0.9840 & 0.0015 & $345 \pm 30$ & -35.0 \\
\hline 35 & 0.0148 & 0.0000 & 0.0533 & 0.0011 & 0.0187 & 0.0036 & 0.0000 & 0.9853 & 0.0000 & $391 \pm 64$ & -29.1 \\
\hline 36 & 0.0449 & 0.0003 & 0.0492 & 0.0003 & 0.0239 & 0.0053 & 0.0002 & 0.9803 & 0.0003 & $462 \pm 131$ & -22.3 \\
\hline 37 & 0.0670 & 0.0000 & 0.0531 & 0.0022 & 0.0147 & 0.0035 & 0.0000 & 0.9850 & 0.0008 & $475 \pm 54$ & -20.4 \\
\hline 38 & 0.0179 & 0.0000 & 0.0513 & 0.0036 & 0.0219 & 0.0001 & 0.0011 & 0.9822 & 0.0007 & $417 \pm 29$ & -27.6 \\
\hline 39 & 0.0059 & 0.0000 & 0.0467 & 0.0023 & 0.0162 & 0.0054 & 0.0001 & 0.9814 & 0.0013 & $342 \pm 33$ & -35.1 \\
\hline 40 & 0.0059 & 0.0000 & 0.0467 & 0.0020 & 0.0183 & 0.0026 & 0.0007 & 0.9831 & 0.0016 & $342 \pm 33$ & -35.1 \\
\hline 41 & 0.0070 & 0.0008 & 0.0396 & 0.0034 & 0.0186 & 0.0018 & 0.0001 & 0.9828 & 0.0015 & $352 \pm 17$ & -33.9 \\
\hline 42 & 0.0111 & 0.0012 & 0.0439 & 0.0038 & 0.0151 & 0.0018 & 0.0000 & 0.9849 & 0.0011 & $369 \pm 16$ & -31.1 \\
\hline 43 & 0.0085 & 0.0006 & 0.0477 & 0.0020 & 0.0163 & 0.0045 & 0.0008 & 0.9758 & 0.0004 & $356 \pm 35$ & -32.8 \\
\hline 44 & 0.0051 & 0.0008 & 0.0527 & 0.0027 & 0.0200 & 0.0029 & 0.0000 & 0.9817 & 0.0013 & $339 \pm 28$ & -35.9 \\
\hline
\end{tabular}

*Equilibrium temperatures and oxygen fugacities calculated using geothermometer/oxygen barometer (QUILF) by Frost et al. (1988), Frost and Lindsley (1992) and Lindsley.

Analyses listed in Tab. 4.

both the interoxide and intraoxide exchange reactions were observed in the Kokava Fe-rich gneisses. Ilmenite exsolution lamellae in magnetites have mostly the same composition as solitary large ilmenites. But some ilmen- ite exsolutions do differ, having relatively elevated $X_{\text {hem }}$. The other end-member mole proportions remain the same or become slightly higher. Host magnetite has higher $\mathrm{X}_{\text {usp }}$ than the average derived from homogeneous magnetites. 
Tab. 6 The results of EPMA monazite dating

\begin{tabular}{|c|c|c|c|c|c|c|c|c|c|c|c|}
\hline $\begin{array}{l}\text { inclusion } \\
\text { in }\end{array}$ & comments & Age & $\begin{array}{c}\text { Pb } \\
\text { (wt. \%) }\end{array}$ & $\begin{array}{c}U \\
\text { (wt. \%) }\end{array}$ & $\begin{array}{c}\text { Th } \\
\text { (wt. \%) }\end{array}$ & $\begin{array}{c}Y \\
\text { (wt. \%) }\end{array}$ & $\begin{array}{l}\text { Th } \\
(1 s)\end{array}$ & $\begin{array}{c}U \\
(1 s)\end{array}$ & $\begin{array}{c}\mathrm{Pb} \\
(1 \mathrm{~s})\end{array}$ & Th*1 & Age $(\mathrm{Ma})^{2}$ \\
\hline Grt & A thick light rim & Permian & 0.2866 & 0.5325 & 20.4530 & 0.2129 & 0.0608 & 0.0117 & 0.0070 & 22.18 & $289 \pm 7.9$ \\
\hline Grt & A thick light rim & Carboniferous & 0.2850 & 0.5170 & 19.4528 & 0.2030 & 0.0553 & 0.0107 & 0.0065 & 21.13 & $302 \pm 7.7$ \\
\hline Grt & A dark core & Carboniferous & 0.5454 & 5.0555 & 1.1956 & 0.0300 & 0.0110 & 0.0064 & 0.0048 & 18.08 & $400 \pm 12.6$ \\
\hline Grt & $\mathrm{B}$ broad dark core & Neo-Proterozoic & 0.1063 & 0.2447 & 3.4783 & 0.0534 & 0.0236 & 0.0093 & 0.0057 & 4.29 & $552 \pm 34.3$ \\
\hline Grt & $\mathrm{B}$ broad dark core & Cambrian & 0.1092 & 0.2804 & 3.8314 & 0.0384 & 0.0245 & 0.0094 & 0.0057 & 4.76 & $512 \pm 30.9$ \\
\hline Grt & $\mathrm{C}$ homogeneous core & Permian & 0.2296 & 0.5766 & 19.0415 & 0.1097 & 0.0545 & 0.0108 & 0.0062 & 20.90 & $246 \pm 7.3$ \\
\hline Grt & $\mathrm{C}$ homogeneous core & Permian & 0.2216 & 0.6424 & 19.9504 & 0.0988 & 0.0561 & 0.0110 & 0.0062 & 22.02 & $226 \pm 7.0$ \\
\hline Grt & $\mathrm{C}$ homogeneous core & Carboniferous & 0.2843 & 0.7673 & 17.9655 & 0.1106 & 0.0527 & 0.0112 & 0.0064 & 20.46 & $311 \pm 7.9$ \\
\hline Grt & $\mathrm{C}$ homogeneous core & Permian & 0.2518 & 0.6401 & 20.4826 & 0.1083 & 0.0569 & 0.0110 & 0.0063 & 22.55 & $250 \pm 6.9$ \\
\hline Grt & D broad dark core & Neo-Proterozoic & 0.1879 & 0.3193 & 6.4670 & 1.5438 & 0.0311 & 0.0096 & 0.0061 & 7.52 & $556 \pm 21.0$ \\
\hline Grt & D broad dark core & Cambrian & 0.1762 & 0.3110 & 6.6748 & 1.4596 & 0.0315 & 0.0095 & 0.0060 & 7.70 & $510 \pm 20.1$ \\
\hline Grt & D broad dark core & Neo-Proterozoic & 0.1901 & 0.2869 & 6.4956 & 0.9108 & 0.0311 & 0.0095 & 0.0060 & 7.45 & $568 \pm 21.1$ \\
\hline Grt & $\mathrm{H}$ homogeneous core & Cambrian & 0.1176 & 0.0869 & 4.5706 & 0.2974 & 0.0264 & 0.0089 & 0.0057 & 4.86 & $539 \pm 30.6$ \\
\hline Grt & $\mathrm{H}$ homogeneous core & Ordovician & 0.1047 & 0.0934 & 4.5649 & 0.3328 & 0.0264 & 0.0089 & 0.0057 & 4.87 & $479 \pm 29.9$ \\
\hline Grt & I homogeneous, with Ap & Cambrian & 0.2406 & 0.5573 & 8.9745 & 2.1379 & 0.0394 & 0.0113 & 0.0068 & 10.81 & $496 \pm 16.3$ \\
\hline Grt & J zoned, dark core & Neo-Proterozoic & 0.1996 & 0.7091 & 4.9038 & 2.0624 & 0.0296 & 0.0114 & 0.0066 & 7.26 & $612 \pm 23.9$ \\
\hline Grt & D light thin rim & Carboniferous & 0.1483 & 0.3913 & 8.5671 & 0.4139 & 0.0355 & 0.0099 & 0.0059 & 9.84 & $337 \pm 15.0$ \\
\hline Grt & $\mathrm{K}$ very inhomogeneous flakes & Carboniferous & 0.2680 & 0.6606 & 15.1378 & 0.1246 & 0.0479 & 0.0108 & 0.0063 & 17.29 & $347 \pm 9.3$ \\
\hline Grt & $\mathrm{J}$ light flake at the rim & Carboniferous & 0.2493 & 0.6375 & 13.8178 & 0.3182 & 0.0489 & 0.0116 & 0.0067 & 15.89 & \pm 10.8 \\
\hline Grt & $\mathrm{J}$ dark core & Carboniferous & 0.2255 & 0.4303 & 11.2199 & 0.5255 & 0.0440 & 0.0110 & 0.0067 & 12.63 & $414 \pm 23.5$ \\
\hline Grt & $\mathrm{H}$ the most light flake at rim & Alpine & 0.1590 & 0.6254 & 31.5080 & 0.1028 & 0.0784 & 0.0124 & 0.0066 & 33.51 & $107 \pm 4.7$ \\
\hline Aln & L grey core & Neo-Proterozoic & 0.1048 & 0.2750 & 3.2845 & 1.6449 & 0.0227 & 0.0101 & 0.0062 & 4.19 & $574 \pm 38.2$ \\
\hline Aln & $\mathrm{L}$ grey core & Neo-Proterozoic & 0.1222 & 0.2462 & 4.3055 & 1.4061 & 0.0256 & 0.0101 & 0.0063 & 5.12 & $550 \pm 32.0$ \\
\hline Aln & $\mathrm{L}$ dark phase & Cam & 0.0653 & 0.1070 & 2.5979 & 1.1000 & 0.0208 & 0.0096 & 0.0061 & 2.95 & $513 \pm 52.6$ \\
\hline Aln & L light rim & ferouls & 0.1015 & 0.3378 & 6.0676 & 0.4607 & 0.0298 & 0.0105 & 0.0063 & 7.16 & $336 \pm 21.8$ \\
\hline Grt & M core & Carboniferous & 0.1351 & 0.3938 & 7.2542 & 0.2398 & 0.0325 & 0.0106 & 0.0063 & 8.54 & \pm 18.5 \\
\hline $\mathrm{Ilm}$ & $\mathrm{N}$ core & Carboniferous & 0.1321 & 0.2455 & 7.7421 & 0.1695 & 0.0337 & 0.0103 & 0.0064 & 8.54 & \pm 18.7 \\
\hline Grt & O core & ferous & 0.1584 & 0.4519 & 8.9693 & 0.1943 & 0.0361 & 0.0110 & 0.0066 & 10.44 & +15.8 \\
\hline Grt & P core & $\mathrm{Ca}$ & 0.1392 & 0.6934 & 4.1960 & 1.0045 & 0.0253 & 0.0113 & 0.0063 & 6.48 & +25.1 \\
\hline Mag & $\mathrm{R}$ core & iferous & 0.1244 & 0.2436 & 7.3504 & 1.4107 & 0.0327 & 0.0103 & 0.0063 & 8.14 & \pm 19.4 \\
\hline Mag & $\mathrm{S}$ core & Carboniferous & 0.2646 & 0.5061 & 15.6831 & 1.6604 & 0.0485 & 0.0114 & 0.0069 & 17.33 & $362 \pm 10.1$ \\
\hline Grt & $\mathrm{T}$ core & Devonian & 0.1177 & 0.4441 & 5.4542 & 2.1201 & 0.0284 & 0.0108 & 0.0064 & 6.90 & $399 \pm 23.2$ \\
\hline Grt & $\mathrm{T}$ rim & Carbonifero & 0.1101 & 0.4384 & 5.7572 & 2.3749 & 0.0290 & 0.0108 & 0.0062 & 7.18 & $361 \pm 21.7$ \\
\hline Grt & AA hon & Permian & 0.3057 & 0.8174 & 21.5310 & 0.1091 & 0.0442 & 0.0079 & 0.0029 & 24.18 & $283 \pm 3.2$ \\
\hline Grt & AA light zone & Alp & 0.1463 & 0.7583 & 27.8356 & 0.1169 & 0.0542 & 0.0081 & 0.0028 & 30.26 & $109 \pm 2.2$ \\
\hline Grt & c dark phase & ozoic & 0.3064 & 0.1944 & 12.1393 & 1.4757 & 0.0292 & 0.0064 & 0.0030 & 12.78 & $=6.6$ \\
\hline Grt & litic light phase & oic & 0.4072 & 0.2833 & 16.9054 & 1.2722 & 0.0370 & 0.0068 & 0.0032 & 17.84 & $=5.0$ \\
\hline Grt & $\mathrm{CC}$ core & ozoic & 0.2534 & 0.3349 & 10.7706 & 0.6028 & 0.0265 & 0.0064 & 0.0028 & 11.87 & \pm 6.5 \\
\hline Grt & $\mathrm{CC}$ core & Neo-Proterozoic & 0.2733 & 0.3979 & 10.7641 & 0.6127 & 0.0265 & 0.0065 & 0.0028 & 12.08 & $504 \pm 6.6$ \\
\hline Aln & DD light rim & Carboniferous & 0.0859 & 0.2741 & 5.1104 & 1.3641 & 0.0172 & 0.0063 & 0.0026 & 6.00 & $321 \pm 11.3$ \\
\hline Aln & DD dark phase & Neo-Proterozoic & 0.1042 & 0.2914 & 3.4186 & 1.6875 & 0.0136 & 0.0061 & 0.0026 & 4.38 & $530 \pm 16$ \\
\hline Grt & EE large oolitic, core & & 0.2851 & 0.6974 & 20.4225 & 0.3863 & 0.0425 & 0.0076 & 0.0029 & 22.68 & \pm 3.4 \\
\hline Mag & FF sma & us & 0.2397 & 0.5165 & 14.2706 & 1.5437 & 0.0322 & 0.0069 & 0.0028 & 15.95 & $336 \pm 4.7$ \\
\hline Grt & $G G \mathrm{lig}$ & us & 0.2209 & 0.4434 & 13.4098 & 0.1618 & 0.0312 & 0.0068 & 0.0028 & 14.85 & \pm 5.0 \\
\hline Grt & -Si phase & ne & 0.5858 & 21.2323 & 47.3300 & 0.2216 & 0.0868 & 0.0524 & 0.0033 & 115.28 & $115 \pm 0.9$ \\
\hline Grt & II dark core & Neo-Prot & 0.1335 & 0.0985 & 4.3374 & 0.4243 & 0.0154 & 0.0058 & 0.0026 & 4.67 & $635 \pm 15.8$ \\
\hline Grt & II dark core & roterozoic & 0.1547 & 0.1223 & 5.4734 & 0.4682 & 0.0178 & 0.0060 & 0.0027 & 5.88 & $585 \pm 12.9$ \\
\hline Grt & II dark core & Neo-Proterozoic & 0.2095 & 0.2183 & 6.8508 & 0.7941 & 0.0199 & 0.0061 & 0.0027 & 7.58 & $615 \pm 10.3$ \\
\hline Grt & JJ with ap, core & Neo-Proterozoic & 0.1456 & 0.0894 & 5.0785 & 0.2814 & 0.0171 & 0.0060 & 0.0027 & 5.38 & $602 \pm 14.1$ \\
\hline Aln & KK with ap, Th-Si & Alpine & 0.0240 & 0.1271 & 5.9946 & 0.0997 & 0.0187 & 0.0060 & 0.0025 & 6.40 & $84 \pm 9.2$ \\
\hline Grt & , core & & & 0.2565 & 8.0199 & 1.8743 & 0.0223 & 0.0064 & 0.0027 & 8.85 & +8.0 \\
\hline Grt & $\mathrm{MM} \mathrm{C}$ & erozoic & 0.2 & & 7.2 & 06 & 0.0208 & 0.0 & 28 & 7.92 & $=10.3$ \\
\hline Grt & MM lig & rous & & 0.4262 & 9.1346 & 0.3026 & 0.0238 & 0.0065 & 0.0027 & 10.52 & $359 \pm 6.7$ \\
\hline Grt & MM very large, dark core & zoic & 0.1989 & 0.3632 & 6.8768 & 1.5635 & 0.0203 & 0.0065 & 0.0028 & 8.08 & $548 \pm 9.7$ \\
\hline Grt & large, dark core & Neo-Proterozoic & 0.1961 & 137 & 6.9972 & 0.9450 & 0.0204 & 0.0064 & 0.0028 & 8.03 & \pm 9.6 \\
\hline Grt & MM very large, light rim & Carboniferous & 0.1619 & 0.4054 & 8.9782 & 0.5550 & 0.0238 & 0.0066 & 0.0027 & 10.30 & $352 \pm 6.9$ \\
\hline Grt & MM very large, light rim & Carboniferous & 0.1528 & 0.4067 & 8.6798 & 0.5635 & 0.0233 & 0.0066 & 0.0027 & 10.00 & $342 \pm 7.1$ \\
\hline Grt & MM very large, light rim & Carboniferous & 0.1620 & 0.4177 & 9.7043 & 0.5453 & 0.0251 & 0.0067 & 0.0027 & 11.06 & $328 \pm 6.5$ \\
\hline Aln, Grt & NN inhomogeneous, light & Permian & 0.2957 & 0.8371 & 18.6595 & 0.1250 & 0.0396 & 0.0078 & 0.0030 & 21.38 & $310 \pm 3.7$ \\
\hline Aln, Grt & NN inh & & & & 21.7470 & & & & 0.0029 & & $=3.2$ \\
\hline Aln, Grt & $\mathrm{NN}$ inhomogeneous, light & & 0.2811 & 0.7829 & 18.2121 & 0.1189 & 0.0389 & 0.0077 & 0.0029 & 20.75 & \pm 3.7 \\
\hline Grt & OO core & Neo-Prote & 0.1098 & 0.0989 & 4.8486 & 0.3000 & 0.0166 & 0.0059 & 0.0026 & 5.17 & $473 \pm 13.7$ \\
\hline Grt & OO core & Neo-Proterozoic & 0.1067 & 0.1090 & 4.6485 & 0.3367 & 0.0162 & 0.0059 & 0.0026 & 5.01 & $475 \pm 14$ \\
\hline Aln & PP core & Neo-Proterozoic & 0.1951 & 0.5633 & 5.5645 & 1.9134 & 0.0179 & 0.0068 & 0.0028 & 7.43 & $585 \pm 10.5$ \\
\hline Aln & PP core & Neo-Proterozoic & 0.1559 & 0.3892 & 4.6959 & 2.0175 & 0.0163 & 0.0064 & 0.0027 & 5.99 & $580 \pm 12.6$ \\
\hline Grt & RR large & Neo-Proterozoic & 0.0861 & 0.1977 & 2.7201 & 2.5434 & 0.0125 & 0.0060 & 0.0026 & 3.38 & $568 \pm 21.3$ \\
\hline Grt & RR large, light rim & Carboniferous & 0.1041 & 0.2179 & 6.1090 & 1.9971 & 0.0189 & 0.0062 & 0.0027 & 6.82 & $342 \pm 10.1$ \\
\hline Grt & RR large, light rim & Co & 0.2146 & 0.3830 & 11.4210 & 1.4394 & 0.0279 & 0.0067 & 0.0028 & 12.67 & $379 \pm 6.0$ \\
\hline Grt & SS dark core & Neo-Proterozoic & 0.1701 & 0.3609 & 5.0817 & 0.4754 & 0.0170 & 0.0064 & 0.0027 & 6.28 & $603 \pm 12.3$ \\
\hline Grt & SS light rim & Carboniferous & 0.1774 & 0.4360 & 9.9089 & 0.0820 & 0.0254 & 0.0067 & 0.0027 & 11.33 & $350 \pm 6.4$ \\
\hline Grt & TT inhomogeneous large & Carboniferous & 0.3324 & 0.5809 & 21.1785 & 0.2386 & 0.0437 & 0.0074 & 0.0030 & 23.07 & $323 \pm 3.5$ \\
\hline Grt & TT inhomogeneous large & Carboniferous & 0.1935 & 0.5242 & 11.0602 & 0.1126 & 0.0273 & 0.0069 & 0.0028 & 12.77 & $339 \pm 5.7$ \\
\hline
\end{tabular}

${ }^{1} \mathrm{Th} *$ includes Th plus equivalent of $\mathrm{U}$. The same letters in the comment denote individual monazite grains.

${ }^{2}$ The ages were calculated after Montel et. al. (1996). 
The origin of Fe-rich gneisses from Kokava (Western Carpathians)

Tab. 7 Representative electron microprobe analyses of monazites used for dating

\begin{tabular}{|c|c|c|c|c|c|c|c|c|c|c|c|c|c|c|c|c|c|}
\hline & $\mathbf{A}^{*}$ & A & $\mathrm{C}$ & C & D & D & D & D & E & G & H & H & $\mathbf{L}$ & M & $\mathbf{N}$ & O & T \\
\hline $\mathrm{iO}_{2}$ & & & 3.82 & 1.20 & & & & & & 1.07 & & & & & & 1.56 & \\
\hline${ }_{2} \mathrm{O}_{3}$ & 00 & 00 & 0.07 & 0.00 & 0.00 & 0.00 & 0.00 & 0.00 & 0.00 & 0.00 & 0.00 & 0.00 & 0.00 & .00 & 0.00 & 0.00 & .00 \\
\hline $\mathrm{a}_{2} \mathrm{O}_{3}$ & .14 & 10.26 & 11.21 & 1.16 & 15.16 & 14.77 & 5.04 & 6 & 13.39 & 0 & 7 & 68 & 26 & 59 & 18 & .62 & 84 \\
\hline $\mathrm{e}_{2} \mathrm{O}_{3}$ & 21.20 & 1.69 & 19.77 & 23.10 & 28.55 & 28.42 & 28.40 & 29.03 & 29.77 & 30.20 & 32.09 & 31.82 & 29.91 & 30.64 & 30.22 & 28.71 & 24.12 \\
\hline${ }_{2} \mathrm{O}_{3}$ & 2.43 & 77 & 90 & .79 & 2.96 & 3.03 & 3.08 & 3.27 & 3.26 & 3.49 & 3.71 & 3.74 & 3.01 & 01 & 3 & 3.08 & 2.46 \\
\hline $\mathrm{d}_{2} \mathrm{O}_{3}$ & 9.39 & 10.22 & 10.26 & 10.22 & 9.15 & 9.33 & 8.86 & 11.18 & 11.26 & 12.29 & 10.87 & 11.04 & 10.52 & 10.99 & 11.06 & 11.47 & 80 \\
\hline $\mathrm{n}_{2} \mathrm{O}_{3}$ & 1.77 & 87 & 1.70 & 1.69 & 1.44 & 1.43 & 1.21 & 1.67 & 1.70 & 2.13 & 77 & 1.58 & 1.49 & & .65 & 1.96 & .51 \\
\hline $\mathrm{u}_{2} \mathrm{O}_{3}$ & 0.03 & 0.12 & 0.00 & 0.00 & 0.11 & 0.20 & 0.20 & 0.00 & 0.00 & 0.08 & 0.00 & 0.00 & 0.15 & 0.06 & 0.00 & 0.00 & 0.07 \\
\hline $\mathrm{d}_{2} \mathrm{O}_{3}$ & 1.5 & 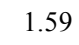 & 1.23 & 1.29 & 1.52 & 1.44 & 1.46 & 1.55 & 1.35 & 1.63 & 1.36 & 1.26 & 1.52 & & 1.57 & 1.67 & 1.47 \\
\hline $\mathrm{b}_{2} \mathrm{O}_{3}$ & 0.05 & 0.04 & 0.00 & 0.03 & 0.09 & 0.13 & 0.06 & 0.09 & 0.06 & 0.03 & 0.10 & 0.02 & 0.00 & 0.04 & 0.00 & 0.01 & 0.05 \\
\hline $\mathrm{y}_{2} \mathrm{O}_{3}$ & 0.20 & 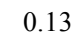 & 0.17 & 0.10 & 0.40 & 0.32 & 0.38 & 0.19 & 0.02 & 0.02 & 0.18 & 0.20 & 0.29 & 0.19 & 0.02 & 0.05 & .43 \\
\hline $\mathrm{O}_{3}$ & 0.25 & & 0.23 & 0.24 & 0. & & & & & & & 6 & & & & 0 & \\
\hline $\mathrm{r}_{2} \mathrm{O}_{3}$ & 0 & & 0.0 & 0.00 & 0.0 & 0 & & 0.00 & 0.0 & 0. & 0 & 0.01 & & & 0.00 & 0.00 & 0.06 \\
\hline $\operatorname{Tm} \Omega$ & & & 0 & 0.00 & & & & & & & & & & & & & \\
\hline $\mathrm{Kb}_{2} \mathrm{O}_{3}$ & 0.06 & 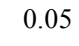 & 0.0 & 0.01 & 0.0 & 0.08 & 0. & 0.00 & 0.0 & 0. & 0 & 0.01 & 0.19 & 0 & 0.03 & 0.03 & 0.11 \\
\hline $\mathrm{Lu}_{2} \mathrm{O}_{3}$ & 0 & & 0.0 & 0.04 & & & & & & & & & & & & 0.00 & 0.00 \\
\hline $\mathrm{b}$ & & & & 032 & & & & & & & & & & & & 0.16 & 0.31 \\
\hline $\mathrm{ThO}_{2}$ & 23.27 & 22.14 & 22.70 & 20.44 & 7.36 & 7.60 & 7.39 & 9.75 & 9.73 & 6.56 & 5.20 & 5.19 & 2.96 & 6.90 & 8.26 & 8.81 & 17.85 \\
\hline 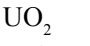 & 061 & & 0.73 & 0.87 & 0.3 & 0.3 & 0. & 0.45 & 0.41 & 0.20 & 0 & 0.11 & 0.12 & & 0.45 & 0.28 & 0.58 \\
\hline $\mathrm{Y}_{2} \mathrm{O}_{3}$ & 0.27 & 0.26 & 0.13 & 0.14 & 1.96 & 1.85 & 1.16 & 0.53 & 0.32 & 0.15 & 0.38 & 0.42 & 1.40 & 59 & 0.31 & 0.22 & 2.11 \\
\hline $\mathrm{P}_{2} \mathrm{O}_{5}$ & 20.94 & 21.66 & 21.23 & 21.85 & 28.50 & 27.68 & 28.04 & 25.72 & 25.97 & 26.53 & 27.04 & 26.90 & 28.58 & 27.25 & 25.99 & 27.83 & 21.45 \\
\hline $\mathrm{aO}$ & 0.72 & 0.73 & 0.98 & 0.74 & 1.40 & 1.38 & 2.12 & 0.53 & 0.67 & 0.51 & 0.07 & 0.07 & 1.75 & 0.49 & 0.53 & 1.40 & 0.45 \\
\hline $\mathrm{As}_{2} \mathrm{O}_{5}$ & 0.15 & 0.14 & 0.16 & 0.13 & 0.13 & 0.11 & 0.13 & 0.15 & 0.18 & 0.15 & 0.15 & 0.13 & 0.16 & 0.15 & 0.13 & 0.13 & 0.17 \\
\hline $\mathrm{FeO}$ & & & & & & & & & & & & & 0.83 & 0.66 & 1.15 & 1.09 & 1.06 \\
\hline tal & & 15 & 39 & 25 & 8 & 29 & 3.85 & 88 & 0.19 & 92 & & 5 & 99.47 & 0.80 & 100.26 & 1.81 & 100.36 \\
\hline
\end{tabular}

*Letters correspond to those in Tab. 6.

A change in the chemical composition of the exsolved magnetite is reflected in the highest recorded temperatures, exceeding $380^{\circ} \mathrm{C}$. The two points with very high $\Delta \log \mathrm{fO}_{2}(\mathrm{FM} \alpha \mathrm{Q})$ of 3.4 and 4.7 , and the temperatures of 462 and $475^{\circ} \mathrm{C}$, fall outside the general trend. This is considered to be the consequence of a local disequilibrium, having no real significance. Consistently lower temperatures were found in other magnetites with ilmenite exsolutions and forming neighbouring homogeneous magnetite-ilmenite pairs. Almost identical composition and comparable $T-\log \mathrm{fO}_{2}$ trend indicate uniform style of subsolidus re-equilibration with the temperature decrease. The temperatures below $\sim 350^{\circ} \mathrm{C}$ showing unrealistic drop in oxygen fugacity might be due to the low-temperature magnetic phenomena not involved in the calculation or the extrapolation to low temperatures, far below the experimental conditions.

During the low-temperature re-equilibration in a closed Fe-Ti system, where the oxygen fugacity is controlled solely by $\mathrm{Fe}^{3+}$ and $\mathrm{Ti}$ exchange reaction between magnetite and ilmenite, the mineral proportions can influence the $T-\log f_{2}$ trend (Frost et al. 1988). If the volume of magnetite is much higher than that of ilmenite, the interoxide re-equilibration follows the isopleths of magnetite and consequently the ilmenite composition changes to buffer the exchange reactions. On the contrary, if ilmenite dominates over magnetite, the ilmenite composition remains nearly constant and it is the magnetite chemistry which changes dramatically.

The thermobarometric estimates for the partial anatexis in the South Veporic Unit using mineral stability calculations give $c$. $680-730^{\circ} \mathrm{C}$ resp. $650-700^{\circ} \mathrm{C}$ and $400-600$ $\mathrm{MPa}$; retrograde isobaric metamorphic conditions drop to 550-600 ${ }^{\circ} \mathrm{C}$ (Siman et al. 1996; Siman and Janák 2005). Main metamorphic rock types, except for anatexites, regionally metamorphosed under the amphibolite-facies conditions, did not record temperatures above $600{ }^{\circ} \mathrm{C}$. Similarly Korikovskij et al. (1989) reported temperature estimates of $550-580^{\circ} \mathrm{C}$, Radvanec (2000) $550-600^{\circ} \mathrm{C}$ and Kováčik (2000) $600 \pm 50^{\circ} \mathrm{C}$.

During a slow cooling, commonly at temperatures of $c$. $450-500^{\circ} \mathrm{C}$, the titanomagnetite exsolved a Ti-rich phase. The presence of titanomagnetite with ilmenite exsolutions thus defines a minimum temperature of the regional metamorphism. Advancing subsolidus re-equilibration upon cooling changed the original composition of $\mathrm{Fe}-\mathrm{Ti}$ oxides. The best preserved composition in magnetite with ilmenite intergrowths was at $380-417^{\circ} \mathrm{C}$ and $\log f \mathrm{O}_{2}$ corresponding to FM $\alpha \mathrm{Q}$ buffer (Fig. 3, Tab. 5). Gradual bending of $\mathrm{fO}_{2}$ trend towards more reduced conditions relative to the FM $\alpha \mathrm{Q}$ buffer can be explained by reequilibration of the prevalent magnetite with subordinate 
ilmenite during cooling. Tracing back the model trend of re-equilibration to the higher temperatures, at about $500-600^{\circ} \mathrm{C}$, the estimated interval of oxygen fugacity is between +1 and $+3 \Delta \log f_{2}(\mathrm{FM} \alpha \mathrm{Q})$ (Fig. 3). Oxygen fugacity above the FM $\alpha \mathrm{Q}$ buffer was most probably dominant during the amphibolite-facies regional metamorphism. Comparable fairly oxidized conditions were documented by Mücke (2003) for the magnetite-silicate and oxidic facies of the Precambrian iron ores.

\subsection{Decomposition of allanite}

Although forming apparently euhedral crystals in the Fe-rich gneisses, the original allanite was, according to chemical analyses, entirely replaced by a mixture of various secondary phases (Tab. 3). Composition of newly formed minerals reflects a gradually changing degree of allanite breakdown. Examples of allanite decomposition in various rock types document a wide variability of reactions producing numerous minerals (Meintzer and Mitchell 1998; Smith et al. 2002). In our case, usually $\mathrm{Ca}$ and REE are preferably released from the allanite structure to be accumulated in cerianite in the nearby cracks. Increased amounts of cerianite forming local accumulations patches up to $25 \mu \mathrm{m}$ in size in Fe-rich gneisses indicate the intensity of the allanite breakdown. Finally, the overall decomposition may have led to the complete replacement by a mixture of clay minerals with iron oxides or hydroxides. Analyses in Tab. 3 include alteration products ranging from a prevalence of clay minerals - most probably halloysite (analysis 4) to iron oxide/hydroxide phases - most probably goethite (analysis 5). Conspicuously, the alteration leaves the immobile Th in alteration products, preferably accumulating in Ferich phases. With increasing $\mathrm{Si}-\mathrm{Al}$ corresponding to the clay component, the Th concentration gradually lowers. Notable are the high $\mathrm{P}_{2} \mathrm{O}_{5}$ concentrations (analyses 1a-b), which decrease with increasing $\mathrm{SiO}_{2}$. Extra phosphorus could have been transported by external hydrothermal fluids; alternatively the allanite could have embayed some P-rich mineral which was completely consumed together with the host allanite. On the other hand, the allanite breakdown produced secondary monazite and other phases during weathering.

One allanite crystal contained locally numerous small cracks which terminated at the grain boundaries, resembling somewhat a radial pattern. The cracks were highlighted by cerianite in BSE images. The cracks might point to an early metamictization since the formation of cerianite should have preceded the origin of clay minerals and Fe-oxides/hydroxides.

Monazite inclusions hosted by larger allanites evoke a breakdown of monazite described by Finger et al. (1998) with Broska and Siman (1998). Recent studies proved the presence of monazites with apatite and allanite coronas in the nearby orthogneisses at the slopes of the Kotoška Hill, $15 \mathrm{~km} \mathrm{NW}$ of the Fe-rich gneisses (Finger et al. 1998). In spite of this, the following arguments point against the monazite breakdown scenario: 1 - monazites show sharp contacts with allanite; 2 - apatite and monazite in one inclusion formed ovoid shapes with sharp boundaries; the same ovoid inclusions were found in almandine; 3 - allanites occur as large prismatic crystals, the radial pattern formed by numerous small crystals is missing and $4-$ the typical coronal texture is absent. These facts suggest that monazites were accidentally incorporated in the cores of newly formed allanites.

\subsection{Evolution of the Kokava ironstones}

Based on the study of Precambrian sedimentary iron formations (from 5 continents and 45 localities), Mücke (2003) distinguished the following groups: a) oxidic facies (with mineral assemblage magnetite, martite, hematite and quartz), b) silicate facies without magnetite, c) magnetite-silicate facies and d) carbonaceous facies. Oxidic facies is characterized by the sum of $\mathrm{Fe}_{2} \mathrm{O}_{3}+\mathrm{SiO}_{2}$ $>90$ wt. \%, while the other groups show lower sums of 75.5-86.1 wt. \%. Kokava Fe-rich gneisses with $\mathrm{Fe}_{2} \mathrm{O}_{3}+$ $\mathrm{SiO}_{2} \sim 84.1$ wt. \%, increased $\mathrm{Al}_{2} \mathrm{O}_{3}$ and $\mathrm{CO}_{2}$ and according to the modal composition belong to the iron formations, more exactly to the magnetite-silicate facies. The occurrence of allanite, which is a mineral not typical of the iron formations, together with high $\mathrm{P}_{2} \mathrm{O}_{5}(>0.7 \mathrm{wt}$. $\%$, in our sample 1.05 wt. \%, Tab. 1), argue against this. Similar rocks with uncertain origin should be classified as the Itakpe Hill type (Mücke 2003).

Low bulk $\mathrm{Ca}$ contents expressed by the absence of grossularite and elevated $\mathrm{P}_{2} \mathrm{O}_{5}$ concentrations (Tab. 1) argue for sedimentary oolitic ironstones as a pre-metamorphic protolith (Zoubek and Nemčok 1951; Korikovskij et al. 1989; Kováčik 2000). Elevated $\mathrm{P}_{2} \mathrm{O}_{5}$ contents were interpreted to have resulted from reworking and recrystallization of organic calcareous shells and microorganisms. We share the same opinion. However, the finding of some ovoid apatite and monazite inclusions in almandine we connect rather with their detrital-clastogene origin (Fig. $2 \mathrm{~g}$ ). The same shapes (strongly rounded, nearly spherical) are typical of all detrital zircons and most of single grain monazites included in almandine. A typical magmatic zoning was preserved in zircons as corroded cores or oscillatory zoning.

The oldest monazites are of Late Proterozoic to Early Palaeozoic age (670-470 Ma). These data are in accord to recently published dating results of igneous, metamorphic and/or detrital zircons not only from neighbouring area but also from wide regions in the Western Carpathians (Gaab et al. 2005; Kohút et al. 2008; Putiš et al. 2008, 2009). The variability of the ages (200 my absolute in- 
terval) and the chemical differences only emphasize their detrital origin. The old ages from the Late Proterozoic to the Early Ordovician mostly coincide with those from pan-African orogenic belt and associated granitoids and metamorphic rocks. The age spectrum of $660-500 \mathrm{Ma}$ in detrital zircons is typical of the northern peri-Gondwana margin (Nance and Murphy 1996). Continental islandarc magmatism characteristic of the northern edge of Gondwana is ascribed to a continent break-up during the rifting and opening the Rheic Ocean, and formation of the Avalonia and Armorica microcontinents, respectively Hunic Superterrane formation (Stampfli and Borel 2002; von Raumer et al. 2002).

The likely sedimentation environment of ferruginous sediments, the presumed protolith of the Kokava Ferich gneisses, was some type of a deltaic system, partly closed bay or lagoon in a near-shore zone (e.g. Kováčik 2000). The volcanic activity most probably contributed to sedimentation of oolitic chamosite iron ores. Part of the metasediments in the Western Carpathians was deposited from the Early Ordovician up to the Carboniferous times. Phanerozoic oolitic ironstones of the world formed in Ordovician or Devonian (e.g. Petránek and Van Houten 1977). However, Middle Ordovician period (c. $470 \mathrm{Ma}$ ) is generally attributed to the opening of Prototethys (Stampfli and Borel 2002) accompanying the Avalonia and Armorica detachment from the northern Gondwana. In the Western Carpathians, the Ordovician sediments are known only from the Gelnica Group of the Gemeric Unit (Snopko 1967). Contrary to this, the magmatic products preserved in the southern part of the Veporic Unit indicate the evolution under more continental conditions. In spite of the limited number of stratigraphic studies in the Western Carpathians, we attribute more significance to younger sedimentation during Silurian to Devonian (Čorná and Kamenický 1976). A wider extent of the remnants of volcano-sedimentary metamorphic sequences was recently proved, including a Lahn-Dill ore deposit in the Slovakian part of the Western Carpathians (Kohút 2006; Kohút et al. 2006). Taken together, we prefer the Devonian rather than the Ordovician period for the sedimentation of the Kokava ironstones. The presence of detrital monazites with ages $\sim 470$ Ma represents the upper age constraint for the sedimentation of the Fe-gneissic protolith and is not at odds with the Devonian age for the oolitic sedimentation.

The best timing of the main Variscan continental collision associated with a high-temperature metamorphism is c. 370-350 Ma according to $\mathrm{U}-\mathrm{Pb}$ zircon dating (e.g. Michalko et al. 1998; Finger et al. 2003). After the termination of collisional movements, the bulk of granitic S-type intrusions was emplaced during Meso-Variscan orogeny, at 350-346 Ma (e.g. Finger et al. 2003).

Our EPMA monazite dating of the Kokava Fe-rich gneisses shows an increase in number of ages between
360-340 Ma and a younger gradual decrease up to the $310 \mathrm{Ma}$ (Fig. 4). The resulting age $342 \pm 2 \mathrm{Ma}$ is almost the same as that from surrounding orthogneisses, paragneisses and granitic rocks pointing to their synchronous metamorphic/magmatic evolution since the Early Carboniferous (Hraško et al. 2005; Kováčik et al. 2005; Siman and Janák 2005).

The presence of Late Carboniferous to Permian ages in the Kokava Fe-rich gneisses is probably the last recorded event in monazites, indicating the termination of the Variscan orogeny. The lowest possible temperatures of monazite crystallization are $c .400{ }^{\circ} \mathrm{C}$ (e.g. Townsend et al. 2001) and this may indicate possible survival of relatively elevated temperatures long after the MesoVariscan-Early Carboniferous granite emplacement, or it reflects thermal effects of more distal Late Carboniferous to Permian granitic intrusions, marking the onset of the Early Alpine continental rifting.

Alpine ages constrained by monazite geochronology in the South Veporic Unit are very rare, however a monazite grain included in quartz from the Liešnica Valley orthogneiss having a $129 \mathrm{Ma}$ in core and $77 \mathrm{Ma}$ in rim was identified by Siman and Janák (2005). In spite of scarcity of the age data, the weak thermal effects of Alpine orogeny are recorded by chloritization, silicification, carbonatization and sulphide impregnations of the studied rocks (e.g. Kováčik 2000). The Fe-rich gneisses from Kokava were only slightly influenced by Alpine hydrothermal alterations. Biotites underwent only slight chloritization, lowering the analyses totals and releasing some K from the structure. Barite and sulphides crystallized in some weakened zones in altered allanites.

\section{Conclusions}

Detailed geochemical, mineralogical and geochronological study of the Fe-rich gneisses from the vicinity of Kokava nad Rimavicou brought the following results:

- Whole rock chemistry, and in particular lack of $\mathrm{CaO}$, $\mathrm{MgO}$ and somewhat elevated $\mathrm{P}_{2} \mathrm{O}_{5}$ contents, rule out the outdated skarn hypothesis, confirming an origin as metamorphosed sedimentary ironstones.

- The mineral assemblage almandine + magnetite + grunerite + ilmenite + quartz + apatite + allanite + zircon + monazite reflects mostly the Variscan metamorphic overprint and following cooling.

- The temperatures calculated from the Fe-Ti oxides are typically subsolidus. The highest determined temperatures recorded in the magnetite with exsolved ilmenite lamellae fall in the interval $380-417^{\circ} \mathrm{C}$. Re-equilibration of the $\mathrm{Fe}$ - $\mathrm{Ti}$ oxides to lower temperatures is interpreted in terms of slow cooling at the retrograde stage. Oxygen fugacity according to $\mathrm{Fe}-\mathrm{Ti}$ 
equilibria at $400^{\circ} \mathrm{C}$ would correspond to the $\mathrm{FM} \alpha \mathrm{Q}$ buffer (moderate oxidation conditions). Tracing back the $\mathrm{Fe}-\mathrm{Ti}$ compositions to the higher temperatures - up to $500-600{ }^{\circ} \mathrm{C}$ - the prevalence of magnetite over ilmenite would shift the $-\Delta \log f \mathrm{O}_{2}$ to higher values $(+1$ to +3 ) if compared with the FM $\alpha \mathrm{Q}$ buffer. These redox conditions are reasonable for the amphibolite-facies metamorphism.

- Electron-microprobe age data reveal an existence of four different monazite populations: i) $670-470 \mathrm{Ma}$ representing detrital grains derived mostly from the Gondwanan sources; ii) $360-320 \mathrm{Ma}$ interpreted as the Variscan metamorphic growth/re-precipitation with the resulting age of $342 \pm 2$ Ma corresponding to the main Carboniferous orogenic phase in the Western Carpathians; iii) 320-240 Ma reflecting either a gradual cooling/post-peak retrogression close in time to the Permian termination of the Variscan orogeny or thermal effects of a distal Permian granite intrusion emplaced at onset of the Early Alpine continental rifting; iv) indication of Cretaceous ages (110-80 Ma) might document an Alpine overprint common in the SE part of the Veporic Unit.

Acknowledgements. We are indebted to the Martin Kováčik and Lubomír Hraško for valuable information and fruitful discussions. Journal reviews by Prof. J. Zimák and an anonymous reviewer are gratefully acknowledged. The authors wish to thank to handling editor Prof. M. Štemprok, Dr. V. Janoušek and Dr. S. Vrána for suggesting improvements to the manuscript and editorial assistance. This study was supported by Grant APVV549-07 and by project VEGA No 1/1026/04.

\section{References}

Bezák V, Hraško L, Kováčik M, Madarás J, Siman P, Pristaš J, Dublan L, KoneČnÝ V, Plašienka D, VozÁrová A, Kubeš P, Švasta J, Slavkay M, Liščák P (1999) Explanations to the geological map of the Slovenské Rudohorie Mts. -Western part, 1 : 50 000. Geological Survey of Slovak Republic ( ̌́GÚDŠ), pp 1-178 (in Slovak, English summary)

Broska I, Siman P (1998) The breakdown of monazite in the West-Carpathian Veporic orthogneisses and Tatric granites. Geol Carpath 49: 161-167

Buddington AF, Lindsley DH (1964) Iron-titanium oxide minerals and synthetic equivalents. J Petrol 5: 310-357

ČORnÁ O, Kamenický L (1976) Ein Beitrag zur Stratigraphie des Kristallinikum der Westkarpaten auf Grund der Palynologie. Geol Zbor Geol Carpath 27: 117-132

Dallmeyer RD, Neubauer F, Handler R, Fritz H, Müller W, PANa D, Putiš M (1996) Tectonothermal evolution of the internal Alps and Carpathians; evidence from ${ }^{40} \mathrm{Ar} /{ }^{39} \mathrm{Ar}$ mineral and whole-rock data. Eclogae Geol Helv 89: 203-227

Finger F, Broska I, Roberts MP, Schermaier A (1998) Replacement of primary monazite by apatite-allaniteepidote coronas in an amphibolite facies granite gneiss from the eastern Alps. Amer Miner 83: 248-258

Finger F, Broska I, Haunschmid B, HrašKo L', Kohút M, Krenn E, Petrík I, Riegler G (2003) Chemical Th(U)-Pb dating of accessory monazites from Western Carpathians basement granitoids by means of the electron microprobe. Int J Earth Sci 92: 86-98

FLORAN RJ, PAPIKE JJ (1978) Mineralogy and petrology of the Gunflint iron formation, Minnesota-Ontario: correlation of compositional and assemblage variations at low to moderate grade. J Petrol 19: 215-288

Frost D, Lindsley DH (1992) Equilibria among Fe-Ti oxides, pyroxenes, olivine, and quartz: part II. Application. Amer Miner 77: 1004-1020

Frost B, Lindsley DH, Andersen D (1988) Fe-Ti oxidesilicate equilibria: assemblages with fayalitic olivine. Amer Miner 73: 727-740

Gaab AS, Poller U, Janák M, Kohút M, Todt W (2005) Zircon $\mathrm{U}-\mathrm{Pb}$ geochronology and isotopic characterization for the pre-Mesozoic basement of the northern Veporic Unit (Central Western Carpathians, Slovakia). Schweiz Mineral Petrogr Mitt 85: 69-88

GaAb A, Poller U, Todt W, Janák M (2006) Alpine reworking of Ordovician protoliths in the Western Carpathians: geochronological and geochemical data on the Murán̆ Gneiss Complex, Slovakia. Lithos 87: 261-275

GubAČ J (1957) Paragenesis of the skarn deposit near Kokava nad Rimavicou. Geol Práce, Zpr 11: 89-99 (in Slovak, English summary)

HrašKo L, Madarás J, Németh Z, KováčIK M, Siman P, Demko R, Král J, Maglay J, Šmon L, Nagy A, Vozárová A, Radvanec M, Putiš M (2005) Evaluation of the geologic and ore potential of the Slovenské Rudohorie Mts. - possibilities of their use for the regional development, Part 1 - geology. In: HrašKo L, MADARÁs J, NÉmeth Z (eds) Final report no. 2898 of the Slovak Rudohorie Mts, Western part, with a map 1: 50 000. Open file report, Dionyz Štúr State Institute of Geology, Bratislava, pp 1-404 (in Slovak)

JanÁK M, Plašienka D, Frey M, Cosca M, Schmidt ST, LuptÁK B. MÉres Š (2001) Cretaceous evolution of a metamorphic core complex, the Veporic unit, Western Carpathians (Slovakia): $\mathrm{P}-\mathrm{T}$ conditions and in situ ${ }^{40} \mathrm{Ar} /{ }^{39} \mathrm{Ar}$ UV laser probe dating of metapelites. J Metamorph Geol 19: 197-216

JANÁK M, Méres Š, Ivan P (2007) Petrology and metamorphic $\mathrm{P}-\mathrm{T}$ conditions of eclogites from the northern Veporic Unit (Western Carpathians, Slovakia). Geol Carpath 58: 121-131 
JeřÁbek P, JanÁk M, Faryad SW, Finger F, KoneČnÝ P (2008) Polymetamorphic evolution of pelitic schists and evidence for Permian low-pressure metamorphism in the Vepor Unit, west Carpathians. J Metamorph Geol 26: 465-485

Kimberley MM (1974) Origin of iron ore by diagenetic replacement of calcareous oolite. Nature 250: 319-320

KLEIN C (2005) Some Precambrian banded iron-formations (BIFs) from around the world: their age, geologic setting, mineralogy, metamorphism, geochemistry, and origins. Amer Miner 90: 1473-1499

Klein C, Ladeira EA (2000) Geochemistry and petrology of some Proterozoic banded iron-formations of the Quadrilátero Ferrífero, Minas Gerais, Brazil. Econ Geol 95: 405-427

Kонút M (2006) Do we have a remnant of the Hanseatic Terrane and/or Rheno-Hercynian Ocean in the Western Carpathians? - a case study from the Devonian of the Považský Inovec Mts. Geolines 20: 67-68

Kohút M, KoneČnÝ P, Siman P (2006) The first finding of the iron Lahn-Dill mineralization in the Tatric Unit of the Western Carpathians. Miner Pol Spec Pap 28: 112-114

Kohút M, Poller U, Gurk Ch, Todt W (2008) Geochemistry and $\mathrm{U}-\mathrm{Pb}$ detrital zircon ages of metasedimentary rocks of the Lower Unit, Western Tatra Mountains (Slovakia). Acta Geol Pol 58: 371-384

KoneČnÝ P, Siman P, Holický I, JanÁk M, Kollárová V (2004) Methodics of monazite dating using electron microprobe. Miner Slov 36: 225-235 (in Slovak, English summary)

Korikovskij SP, Dupej J, Boronikhin VA (1989) Fe-rich metasediments from Kokava nad Rimavicou (Veporikum). Miner Slov 21: 251-258 (in Slovak, English summary)

Korikovsky S P, Putiš M, PlašIEnka D (1997) Cretaceous low-grade metamorphism of the Veporic and North-Gemeric zones: a result of collisional tectonics in the central Western Carpathians. In: Grecula P, Hovorka D, Putiš M (eds) Geological Evolution of the Western Carpathians. Miner Slov - Monograph, Bratislava, pp 107-130

KovÁČIK M (2000) Petrogenesis of metamorphosed ironstones near Kokava nad Rimavicou (Veporicum, Western Carpathians). Slovak Geol Mag 6: 367-376

KovÁčIK M, KRÁL J, MALUSKI H (1996) Metamorphic rocks in the Southern Veporicum basement: their Alpine metamorphism and thermochronologic evolution. Miner Slov 28: 185-202 (in Slovak with English summary)

KováčiK M, KonečnÝ P, Kollárová V, Holický I, Siman P (2005) Electron microprobe dating of monazite in basement metamorphites from Kohút Zone of Veporicum and correlation aspects (Western Carpathians). Slovak Geol Mag 11: 91-105

KrÁL J, Frank W, BeZÁK V (1996) The ${ }^{40} \mathrm{Ar} /{ }^{39}$ Ar spectra of hornblende from the amphibole-bearing rocks of the
Veporic unit. Miner Slov 28: 501-513 (in Slovak with English summary)

Lindsley DH, Frost B (1992) Equilibria among Fe-Ti oxides, pyroxenes, olivine, and quartz: Part I. Theory. Amer Miner 77: 987-1003

Lupták B, JanÁk M, Plašienka D, Schmidt ST (2003) Alpine low-grade metamorphism of the Permian-Triassic sedimentary rocks from the Veporic Superunit, Western Carpathians: phyllosilicate composition and "crystallinity" data. Geol Carpath 54: 367-375

Maluski H, Rajlich P, Matte P (1993) ${ }^{40} \mathrm{Ar} /{ }^{39} \mathrm{Ar}$ dating of the Inner Carpathians Variscan basement and Alpine mylonitic overprinting. Tectonophysics 223: 313-337

Meintzer RE, Mitchell RS (1998) The epigene alteration of allanite. Canad Mineral 26: 945-955

Mellon GB (1962) Petrology of Upper Cretaceous oolitic iron-rich rocks from northern Alberta, Econ Geol 57: 921-940

Michalko J, Bezák V, Král J, Huhma H, Mänttäri I, VAASJOKi M, Broska I, HrašKo L', HATÁr J (1998) U/Pb zircon data from the Veporic granitoids (West Carpathians). Krystalinikum 24: 91-104

Montel JM, Foret S, Veschambre M, Nicollet C, Provost A (1996) Electron microprobe dating of monazite. Chem Geol 131: 37-53

MüCKE A (2003) General and comparative considerations of whole-rock and mineral compositions of Precambrian iron-formations and their implications. Neu Jb Geol Paläont, Abh 179: 175-219

MÜCKE A, FARSHAD F (2005) Whole-rock and mineralogical composition of Phanerozoic ooidal ironstones: comparison and differentiation of types and subtypes. Ore Geol Rev 26: 227-262

Nance RD, Murphy JB (1996) Basement isotopic signatures and Neoproterozoic palaeogeography of Avalonian-Cadomian and related terranes in the circum-North Atlantic. In: NAnce RD, Thompson MD (eds) Avalonian and Related Peri-Gondwanan Terranes of the CircumNorth Atlantic. Geological Society of America Special Papers 304: pp 333-346

Petránek J, VAn Houten FB (1977) Phanerozoic ooidal ironstones. Czech Geological Survey Special Papers 7: pp 1-71

Petrík I, KonečnÝ P (2009) Metasomatic replacement of inherited metamorphic monazite in a biotitegarnet granite from the Nízke Tatry Mountains, Western Carpathians, Slovakia: chemical dating and evidence for disequilibrium melting. Amer Miner 94: 957-974

Plašienka D, Grecula P, Putiš M, Hovorka D, Kováč M (1997) Evolution and structure of the Western Carpathians: an overview. In: Grecula P, Hovorka D, Putiš M (eds) Geological Evolution of the Western Carpathians. Miner Slov - Monograph, Bratislava, pp 1-24 
Plašienka D, JanÁK M, Lupták B, Milovský R, Frey M (1999) Kinematics and metamorphism of a Cretaceous core complex: the Veporic Unit of the Western Carpathians. Phys Chem Earth (A) 24: 651-658

Putiš M, Kotov AB, Korikovsky SP, Salnikova EB, Yakovleva SZ, Berezhnaya NG, Kovach, VP, Plotkina JV (2001) U-Pb zircon ages of dioritic and trondhjemitic rocks from a layered amphibolitic complex crosscut by granite vein (Veporic basement, Western Carpathians). Geol Carpath 52: 49-60

Putiš M, Sergeev S, Ondrejka M, Larionov A, Siman P, SPIŠIAK J, UHER P, PADERIN I (2008) Cambrian-Ordovician metaigneous rocks associated with Cadomian fragments in the West-Carpathian basement dated by SHRIMP on zircons: a record from the Gondwana active margin setting. Geol Carpath 59: 3-18

Putiš M, Ivan P, Kohút M, Spišiak J, Siman P, Radvanec M, Uher P, Sergeev S, Larionov A, Méres Š, Demko R, OndREJKA M (2009) Meta-igneous rocks of the WestCarpathians basement as an indicator of Early Paleozoic extension-rifting/breakup events. Bull Soc Géol France 180: 461-471

RADVANEC M (2000) Metapelite, amphibolite schist, an origin of magnetite-graphite mineralization in Veporicum near Kokava nad Rimavicou. Miner Slov 32: 1-16 (in Slovak, English summary)

RAumer JF von, Stampfl, GM, Borel GD, Bussy F (2002) Organization of pre-Variscan basement areas at the northGondwanan margin. Int J Earth Sci 91: 35-52

SimAN P, JANÁK M (2005) Study of orthogneisses and migmatites in the selected areas of Tatric and Veporic units focused on their age and metamorphic evolution. Open file report, Dionyz Štúr State Institute of Geology, Bratislava, pp 1-52 (in Slovak)

Siman P, Johan V, Ledru P, Bezák V, Madarás J (1996) Deformation and $\mathrm{P}-\mathrm{T}$ conditions estimated in "layered migmatites" from southern part of Veporicum crystalline basement (Western Carpathians; Slovakia). Slovak Geol Mag 3: 209-214
Slavkay M, BeñKa J, Bezák V, Gargulák M, HrašKo L', Kováčik M, Petro M, VozÁrová A, HrušKovič S, KNÉSL J, Knéslová A, Kusein M, Maťová V, Tulis J (2004) The Ore Deposits on the Slovak Rudohorie Mts. - Part 2. Dionyz Štúr State Institute of Geology, Bratislava, pp 1-283 (in Slovak, English summary)

Smith MP, Henderson P, Jeffries T (2002) The formation and alteration of allanite in skarn from the Beinn an Dubhaich granite aureole, Skye. Eur J Mineral 14: 471-486

SNOPKO L (1967) Lithostratigraphic characterisation of Gelnica Group. Západné Karpaty 7: 103-152 (in Slovak, German summary)

StAMPfli GM, Borel GD (2002) A plate tectonic model for the Paleozoic and Mesozoic constrained by dynamic plate boundaries and restored synthetic oceanic isochrons. Earth Planet Sci Lett 196: 17-33

Sturesson U (2003) Lower Palaeozoic iron oolites and volcanism from a Baltoscandian perspective. Sedim Geol 159: 241-256

Sturesson, U, Dronov A, SaAdre T (1999) Lower Ordovician iron ooids and associated oolitic clays in Russia and Estonia: a clue to the origin of iron oolites? Sedim Geol 123: 63-80

ŠUF J (1938) Report on geological setting and economic mineral deposits in the vicinity of Kokava in Slovakia. Věst Stát geol úst ČSR 14: 91-103

Townsend KJ, Miller CF, D’Andrea JL, Ayers JC, HarRISON TM, COATH DC (2001) Low temperature replacement of monazite in the Ireteba granite, Southern Nevada: geochronological implications. Chem Geol 172: 95-112

VRÁNA S (1966) Alpidische Metamorphose der Granitoiden und der Foederata Serie im Mittelteil der Veporiden. Zborník Geol Vied, Západ Karpaty 6: 29-84

Zoubek V (1936) Bemerkungen über das Kristallin der Westkarpathen. Věst Stát geol úst ČSR 12: 207-239

Zoubek V, Nemčok A (1951) Report on magnetite ore deposit near Kokava n./Rimavicou. Open file report, State Geological Institute, Bratislava, pp 1-13 